\title{
Parkinsonian Beta Oscillations in the External Globus Pallidus and Their Relationship with Subthalamic Nucleus Activity
}

\author{
Nicolas Mallet, ${ }^{1,2 \star}$ Alek Pogosyan, ${ }^{2}$ László F. Márton, ${ }^{1,3}$ J. Paul Bolam, ${ }^{1}$ Peter Brown, ${ }^{2}$ and Peter J. Magill ${ }^{1 \star}$ \\ ${ }^{1}$ Medical Research Council Anatomical Neuropharmacology Unit, University of Oxford, Oxford OX1 3TH, United Kingdom, ${ }^{2}$ Sobell Department of Motor \\ Neuroscience and Movement Disorders, Institute of Neurology, London WC1N 3BG, United Kingdom, and ${ }^{3}$ Neural Systems Research Group, Sapientia- \\ Hungarian University of Transylvania, 540485 Târgu-Mureş, Romania
}

\begin{abstract}
Inappropriately synchronized beta $(\beta)$ oscillations $(15-30 \mathrm{~Hz})$ in the subthalamic nucleus (STN) accompany movement difficulties in idiopathic Parkinson's disease (PD). The cellular and network substrates underlying these exaggerated $\beta$ oscillations are unknown but activity in the external globus pallidus (GP), which forms a candidate pacemaker network with STN, might be of particular importance. Using a clinically relevant rat model of PD, we demonstrate that oscillatory activity in GP neuronal networks becomes excessively and selectively synchronized at $\beta$ frequencies in a spatially widespread and brain state-dependent manner after lesion of dopamine neurons. Although synchronization of GP unit activity increased by almost 100 -fold during $\beta$ oscillations, the mean firing rate of GP neurons decreased compared with controls. Importantly, in parkinsonian animals, two main types of GP neuron were identified according to their distinct and inversely related firing rates and patterns. Moreover, neurons of the same type tended to fire together, with small phase differences, whereas different types of neuron tended not to do so. This functional dichotomy in temporal coupling persisted across extreme brain states, suggesting that maladaptive interactions are dominated by hardwiring. Finally, the precisely timed discharges of GP and STN neurons indicated that rhythmic sequences of recurrent excitation and inhibition in the STN-GP network, and lateral inhibition between GP neurons, could actively support abnormal $\beta$ oscillations. We propose that GP neurons, by virtue of their spatiotemporal synchronization, widespread axon collaterals and feed-back/feed-forward mechanisms, are well placed to orchestrate and propagate exaggerated $\beta$ oscillations throughout the entire basal ganglia in PD.
\end{abstract}

Key words: globus pallidus; subthalamic nucleus; basal ganglia; Parkinson's disease; dopamine; 6-hydroxydopamine

\section{Introduction}

Synchronized neuronal oscillations in the brain have been correlated with distinct behavioral or brain states, suggesting a functional link (Singer, 1999; Buzsáki and Draguhn, 2004). Moreover, it has been proposed that synchronized oscillations provide a mechanism for dynamic, optimal communication and computation within and between dispersed neuronal networks (Engel et al., 2001). If synchronized oscillations are indeed important for normal brain function, then abnormal or uncontrolled synchronization could be disadvantageous or even pathological (Schnitzler and Gross, 2005; Uhlhaas and Singer, 2006). This idea is supported by studies in idiopathic Parkinson's disease (PD) and its animal models, in which loss of midbrain dopamine neurons

\footnotetext{
Received Sept. 3, 2008; revised Nov. 11, 2008; accepted Nov. 13, 2008.

This work was supported by the Medical Research Council (UK), The Dana Foundation, and The Parkinson's Disease Society of the United Kingdom (Grant G-0806). We are grateful to Drs. T. Klausberger, A. Sharott, F. Georges, and I. Bar-Gad for valuable scientific discussions. We also thank E. Norman, C. Francis, K. Whitworth, and B. Micklem ARPS for expert technical assistance.

Correspondence should be addressed to either of the following: Dr. Peter J. Magill or Dr. Nicolas Mallet, Medical Research Council Anatomical Neuropharmacology Unit, University of Oxford, Mansfield Road, Oxford OX1 3TH, UK. E-mail: peter.magill@pharm.ox.ac.uk or nicolas.mallet@pharm.ox.ac.uk.

D01:10.1523/JNEUROSCI.4199-08.2008

Copyright $\odot 2008$ Society for Neuroscience $\quad 0270-6474 / 08 / 2814245-14 \$ 15.00 / 0$
}

induces excessive synchronization of (oscillatory) activity in the basal ganglia (BG) and associated circuits (Nini et al., 1995; Bergman et al., 1998; Boraud et al., 2005; Gatev et al., 2006). In PD patients, synchronization of the oscillatory activities of single neurons and/or neuronal populations in cortex, subthalamic nucleus (STN) and internal pallidum preferentially occurs at beta $(\beta)$ frequencies $(15-30 \mathrm{~Hz})$ (Brown, 2006; Hammond et al., $2007)$. These exaggerated $\beta$ oscillations are reduced during voluntary movements (Kühn et al., 2005; Williams et al., 2005), and are attenuated, together with motor symptoms, by therapeutic interventions (Brown et al., 2001; Levy et al., 2002; Williams et al., 2002). Altogether, these clinical studies suggest that, by inappropriately coordinating neuronal activities, exaggerated $\beta$ oscillations play pathological "antikinetic" roles in PD (Brown, 2006).

The cellular and network mechanisms underlying the generation of these exaggerated $\beta$ oscillations in STN and their propagation through corticobasal ganglia circuits are unknown. However, rigorous definition of their neural substrates is critical for determining their functional significance and for designing strategies to control them for symptomatic benefit. One BG nucleus of potential importance for the generation/propagation of oscillatory activity in these circuits is the external globus pallidus (GP). Indeed, GABAergic GP neurons have intrinsic oscillatory prop- 
erties (Nambu and Llinaś, 1994; Stanford, 2003), and the reciprocal connections between GP and STN could, in theory, generate and maintain low-frequency $(\leq 5 \mathrm{~Hz})$ rhythms in vivo (Plenz and Kital, 1999; Terman et al., 2002). Moreover, GP neurons are in a central position to propagate and synchronize oscillatory activity because they possess extensive local axon collaterals and also project to all other BG nuclei (Kita and Kitai, 1994; Bolam et al., 2000; Sadek et al., 2007).

Here, we test the hypothesis that, in parkinsonism, GP neurons excessively synchronize their activity in the $\beta$-frequency band and, moreover, may orchestrate such overt rhythmic activity in the STN. These issues are not easily explored in PD patients because ipsilateral GP and STN are not usually targeted together for surgical intervention. However, a rodent model of PD, the 6-hydroxydopamine (6-OHDA)-lesioned rat, mimics the findings in patients in that excessive $\beta$ oscillations emerge in the STN and cortex according to ongoing movement, dopamine receptor occupancy (Sharott et al., 2005) and brain state (Mallet et al., 2008). Accordingly, we used 6-OHDA-lesioned rats to investigate the neural basis of abnormal synchronized oscillations in GP and STN after dopamine loss.

\section{Materials and Methods}

Experimental procedures were performed on adult male Sprague Dawley rats (Charles River), and were conducted in accordance with the Animals (Scientific Procedures) Act, 1986 (UK), and with Society for Neuroscience Policies on the Use of Animals in Neuroscience Research.

Electrophysiological recordings. Recordings were made in 18 dopamineintact control rats (288-412 g) and 27 6-OHDA-lesioned rats (285-470 $\mathrm{g}$ at the time of recording). Anesthesia was induced with $4 \% \mathrm{v} / \mathrm{v}$ isoflurane (Isoflo; Schering-Plough) in $\mathrm{O}_{2}$, and maintained with urethane (1.3 $\mathrm{g} / \mathrm{kg}$, i.p.; ethyl carbamate, Sigma), and supplemental doses of ketamine (30 mg/kg, i.p.; Ketaset; Willows Francis) and xylazine (3 mg/kg, i.p.; Rompun, Bayer), as described previously (Magill et al., 2006). All wound margins were infiltrated with the local anesthetic, bupivacaine $(0.75 \%$ $\mathrm{w} / \mathrm{v} ;$ Astra). Animals were then placed in a stereotaxic frame (Kopf). Body temperature was maintained at $37 \pm 0.5^{\circ} \mathrm{C}$ using a homeothermic heating device (Harvard Apparatus). Electrocorticograms (ECoGs), electrocardiographic activity and respiration rate were monitored constantly to ensure the animals' well being (Magill et al., 2006). The ECoG was recorded via a $1 \mathrm{~mm}$ diameter steel screw juxtaposed to the dura mater above the right frontal (somatic sensory-motor) cortex [ $4.5 \mathrm{~mm}$ anterior and $2.0 \mathrm{~mm}$ lateral of bregma (Paxinos and Watson, 1986)], and was referenced against another screw implanted in the skull above the ipsilateral cerebellar hemisphere. Raw ECoG was bandpass filtered (0.3$1500 \mathrm{~Hz},-3 \mathrm{~dB}$ limits) and amplified (2000×; DPA-2FS filter/amplifier; Scientifica) before acquisition. Extracellular recordings of unit activity and local field potentials (LFPs) in the GP were simultaneously made using "silicon probes" (NeuroNexus Technologies). Each probe had two vertical arrays of 16 recording contacts. The arrays were separated by 500 $\mu \mathrm{m}$, and, along each array, the recording contacts were separated by 100 $\mu \mathrm{m}$. Each contact had an impedance of $0.9-1.3 \mathrm{M} \Omega$ (measured at 1000 $\mathrm{Hz}$ ) and an area of $\sim 400 \mu \mathrm{m}^{2}$. The same probe was used throughout these experiments, but it was cleaned after each experiment in a proteolytic enzyme solution (Magill et al., 2006). This was sufficient to ensure that contact impedances and recording performance were not altered by probe use and reuse. Monopolar probe signals were recorded using highimpedance unity-gain operational amplifiers (Advanced LinCMOS; Texas Instruments) and were referenced against a screw implanted above the contralateral cerebellar hemisphere. Probes were advanced into the brain under stereotaxic control (Paxinos and Watson, 1986), at an angle of $15^{\circ}$ to the vertical to maximize the spread of recording contacts across the GP. After initial amplification, extracellular signals were further amplified $(1000 \times)$ and low-pass filtered $(0-6000 \mathrm{~Hz})$ using programmable differential amplifiers (Lynx-8; Neuralynx). The ECoG and probe signals were each sampled at $17.9 \mathrm{kHz}$ using a Power1401 Analog-Digital con- verter and a PC running Spike2 acquisition and analysis software (Cambridge Electronic Design).

The GP was easily distinguished from the striatum in which characteristically low levels of unit activity were observed (Mallet et al., 2005, 2006). Recording locations were additionally verified after the experiments using standard histological procedures (Magill et al., 2006). In some experiments, we simultaneously recorded activity in STN and GP. Unit activity and LFPs were recorded in the STN using silicon probes (as above), or more commonly, using glass electrodes. In the latter case, extracellular recordings of action potentials of STN neurons were made using 15-25 $\mathrm{M} \Omega$ glass electrodes (tip diameter $\sim 1.5 \mu \mathrm{m}$ ), which contained saline solution $(0.5 \mathrm{M} \mathrm{NaCl})$ and Neurobiotin $(1.5 \% \mathrm{w} / \mathrm{v}$, Vector Laboratories). Electrode signals were amplified $(10 \times)$ through the active bridge circuitry of an Axoprobe-1A amplifier (Molecular Devices), ACcoupled, amplified a further $100 \times$ and bandpass filtered at $300-5000 \mathrm{~Hz}$ (DPA-2FS; Scientifica), and finally, sampled as for probe signals (see above). The STN was initially identified by comparison of recorded unit activity with the known characteristic discharges of STN neurons in urethane anesthesia (Magill et al., 2001). Moreover, the recording of activity evoked by bipolar electrical stimulation of the ipsilateral frontal cortex allowed unequivocal targeting of the STN during experiments (Magill et al., 2004).

Activity was recorded, first, during slow-wave activity (SWA), which accompanies deep anesthesia and is similar to activity observed during natural sleep, and second, during episodes of spontaneous "cortical activation," which contain patterns of activity that are more analogous to those observed during the awake, behaving state (Steriade, 2000). It is important to note that the neuronal activity patterns present under this anesthetic regimen may only be qualitatively similar to those present in the unanesthetized brain. Nevertheless, the urethane-anesthetized animal still serves as a useful model for assessing the impact of extremes of brain state on functional connectivity within and between the basal ganglia and cortex (Magill et al., 2006). Cortical activation was occasionally elicited by pinching the hindpaw for $15 \mathrm{~s}$ with serrated forceps that were driven by a standard pneumatic pressure, as described previously (Magill et al., 2006). Note that we did not analyze neuronal activity recorded concurrently with the sensory stimuli. Because the analyzed activity was recorded at least several minutes after the cessation of the brief pinch stimulus, it was also considered as spontaneous. The animals did not exhibit either a marked change in the electrocardiogram or respiration rate, and did not exhibit a hindpaw withdrawal reflex, in response to the pinch. Moreover, withdrawal reflexes were not present during episodes of prolonged cortical activation, thus indicating anesthesia was adequate throughout recordings.

6-Hydroxydopamine lesions of dopamine neurons. Unilateral 6-OHDA lesions were performed on 190-305 g rats, as described previously (Magill et al., 2001). Twenty-five min before the injection of 6-OHDA, all animals received a bolus of desipramine $(25 \mathrm{mg} / \mathrm{kg}$, i.p.; Sigma) to minimize the uptake of 6-OHDA by noradrenergic neurons (Schwarting and Huston, 1996a). Anesthesia was induced and maintained with isoflurane (as above). The neurotoxin 6-OHDA (hydrochloride salt; Sigma) was dissolved immediately before use in ice-cold $0.9 \% \mathrm{w} / \mathrm{v} \mathrm{NaCl}$ solution containing $0.02 \% \mathrm{w} / \mathrm{v}$ ascorbate to a final concentration of $4 \mathrm{mg} / \mathrm{ml}$. Then $3 \mu \mathrm{l}$ of 6-OHDA solution was injected into the region adjacent to the medial substantia nigra $(4.5 \mathrm{~mm}$ posterior and $1.2 \mathrm{~mm}$ lateral of bregma, and $7.9 \mathrm{~mm}$ ventral to the dura) (Paxinos and Watson, 1986). The extent of the dopamine neuron lesion was assessed 14 or $15 \mathrm{~d}$ after 6-OHDA injection by challenge with apomorphine $(0.05 \mathrm{mg} / \mathrm{kg}$, s.c.; Sigma) (Schwarting and Huston, 1996b). The lesion was considered successful in those animals that made $\geq 80$ net contraversive rotations in 20 min. Note that the emergence of exaggerated $\beta$ oscillations after 6-OHDA lesions is not dependent on apomorphine (Sharott et al., 2005). Electrophysiological recordings were performed ipsilateral to 6-OHDA lesions in anesthetized rats 21-45 d after surgery, when pathophysiological changes in the basal ganglia are likely to have leveled out near their maxima (Vila et al., 2000).

Data analysis. Epochs of robust cortical slow-wave activity or cortical activation were selected for analysis according to the previously described characteristics of these brain states (Magill et al., 2001, 2006). 
Analyses were performed on $100 \mathrm{~s}$ of data recorded during robust SWA and $100 \mathrm{~s}$ during cortical activation in each control and 6-OHDAlesioned animal. Population activity, as recorded in ECoGs or as LFPs, was characterized in the frequency domain by deriving autospectra of power by standard discrete Fourier transform-based methods (Spike2). The ECoGs and LFPs were assumed to be realizations of stationary, zeromean time series. Data were windowed with a Hanning filter to control "spectral leakage." For analysis of population activity during SWA, the ECoGs/LFPs were first down-sampled to $256 \mathrm{~Hz}$ (Spike2). The frequency resolution of SWA power spectra was $0.125 \mathrm{~Hz}$. We focused our quantitative comparisons on a frequency range of $\sim 0.4-1.6 \mathrm{~Hz}$, which encompasses the cortical slow oscillation that is dominant during SWA (Magill et al., 2006). The total spectral power within all frequency bins between 0.4 and $1.6 \mathrm{~Hz}$ was calculated. The "dominant frequency" of the ECoGs or LFPs was derived from the frequency bin (within $0.4-1.6 \mathrm{~Hz}$ ) that contained the highest power. For analysis of population activity during cortical activation, the ECoGs/LFPs were also down-sampled to $256 \mathrm{~Hz}$. The frequency resolution of these power spectra was $1 \mathrm{~Hz}$. We focused our comparative analyses on the $\beta$ frequency range $(15-30 \mathrm{~Hz})$, which covers the prevalent cortical rhythms exhibited by 6-OHDA-lesioned animals during the activated brain state (Mallet et al., 2008).

After off-line bandpass filtering $(500-6000 \mathrm{~Hz})$ of the wideband probe signals, putative single-unit activity (signal/noise ratio of $>2.5$ ) was isolated with standard "spike sorting" procedures, including template matching, principal component analysis, and supervised clustering (Spike2). Isolation of single units was verified by the presence of a distinct refractory period in the interspike interval (ISI) histograms. Only neurons in which $<1 \%$ of all ISIs were $<2 \mathrm{~ms}$ were analyzed in this study. For further analysis, single-unit activity was converted so that each spike was represented by a single digital event (Spike2). The mean firing rate of each neuron was calculated from the reciprocal of the mean ISI. The coefficient of variation (CV) of the ISIs, a value used widely as an indicator of regularity in point processes (Johnson, 1996), was also calculated. Auto-correlograms and cross-correlograms were constructed using Spike2 according to standard methods. For the analysis of unit activity during SWA, the correlogram bin size was $10 \mathrm{~ms}$. However, this bin size would give unacceptably poor temporal resolution when testing for synchronized activity at $\beta$ oscillation frequencies. Thus, for the analysis of unit activity during cortical activation, we reduced the correlogram bin size to $2 \mathrm{~ms}$. To test for short-latency, asymmetric temporal relationships between neuron pairs, which may be indicative of monosynaptic interactions, the bin size for cross-correlograms was reduced to $0.5 \mathrm{~ms}$. Significant short-latency temporal interactions were defined as such when peaks and/or troughs within $5 \mathrm{~ms}$ of time $=0$ crossed a threshold of $\pm 2 \mathrm{SD}$ of mean activity (defined at extremes of cross-correlograms).

To quantify the temporal coupling of the spike discharges of pairs of neurons in the frequency domain, we assumed spike trains to be realizations of stationary, stochastic point processes and performed an analysis of coherence between pairs of point processes. The coherence was calculated according to standard methods as described in detail previously (Halliday et al., 1995). Data were windowed with a Hanning filter to control spectral leakage. Significance levels were estimated for coherence spectra according to standard methods (Halliday et al., 1995). For analysis of unit activity during SWA and cortical activation, the coherence analysis was focused on two important frequency bands $(0.4-1.6 \mathrm{~Hz}$ and $15-30 \mathrm{~Hz}$ ). The frequency resolutions for the $0.4-1.6 \mathrm{~Hz}$ and $15-30 \mathrm{~Hz}$ coherence spectra were $0.125 \mathrm{~Hz}$ and $1 \mathrm{~Hz}$, respectively. Significant coherence was defined as such when coherence in at least two consecutive frequency bins (within the ranges of $0.4-1.6 \mathrm{~Hz}$ or $15-30 \mathrm{~Hz}$ ) exceeded the significance level of $p=0.05$. The dominant frequency of such significant coherence was then derived from the frequency bin (within 0.4$1.6 \mathrm{~Hz}$ or $15-30 \mathrm{~Hz}$ ) that contained the highest coherence. For each significantly coherent pair of units, we also calculated the instantaneous phase of the signals at the dominant frequency. Regardless of frequency band analyzed, the bin size for all plots of instantaneous phase values was 10 degrees. Coherence and phase analyses were implemented with custom scripts for use with Spike2 and MATLAB (v7.3; The MathWorks). Note that cross-correlations and coherence/phase estimates were not performed for pairs of units recorded on the same probe contact or glass electrode to avoid "shadowing effects" and similar artifacts (Bar-Gad et al., 2001, 2003). Analysis of each cross-correlogram confirmed that the same GP unit was not recorded on adjacent probe contacts (Mallet et al., 2008).

The slow $(\sim 1 \mathrm{~Hz})$ oscillation that dominates SWA, as recorded in ECoGs or basal ganglia LFPs, does not closely approximate a sinusoidal waveform, and contains active and inactive components of substantially varied shape and duration. This makes detecting the peaks (or troughs) of this oscillation difficult, and also limits the utility of several analyses commonly used for defining the relationships between unit activity and ECoG/LFP oscillations e.g., spike-triggered waveforms or circular statistics. Thus, we used an alternative method of quantifying the temporal relationship between unit activity and the cortical slow oscillation. Single-unit activity was converted so that the "time stamp" of each spike was represented by a single digital event (Spike2). Raw ECoGs were initially down-sampled to $1.79 \mathrm{kHz}$ off-line, high-pass filtered at $0.3 \mathrm{~Hz}$ to remove any slow drift in DC potential, and then bandpass filtered at $0.4-1.6 \mathrm{~Hz}$ to isolate the cortical slow oscillation (MATLAB). The zerovoltage crossings of this filtered ECoG signal were then used to define the start and end points of active (and inactive) components. However, active and inactive components were only considered as such after thresholding for voltage (amplitude difference between components of $\geq 0.3$ $\mathrm{mV}$ ) and power (ratio of component power, which takes into account the signal energy over time, was $>0.15$ ). Thus, only robust slow oscillations were analyzed. Moreover, active components were only accepted if they were preceded or succeeded by an inactive component and vice versa. After defining active or inactive components of ECoGs, coincident spikes were automatically assigned to one of 14 bins ( 7 bins each for active and inactive components; MATLAB). Spike counts per bin across all accepted oscillation components were then normalized (by converting to firing rate) to take into account the variable durations of active and inactive components, and then displayed in an "activity histogram" (see Fig. 1). For statistical definition of relationships between unit activity and the cortical slow oscillation, we used Pearson's $\chi^{2}$ test (Excel; Microsoft Corp.) to assess the goodness of fit of the observed firing, as indicated in the activity histogram, to the expected firing. The null hypothesis, which dictated the expected firing, was that firing during the active component was the same as that during the inactive component, i.e., that unit activity was not modulated in time with the slow oscillation. When expected firing was $<10$, that is, when relatively inactive neurons were tested, we used the nonparametric binomial test (SPSS; SPSS Inc.) instead of the $\chi^{2}$ test. Significance for the $\chi^{2}$ and binomial tests was set at $p<0.05$.

Linear phase histograms were used to quantify the temporal relationships between cortical $\beta$ oscillations, as measured in ECoGs, and singleunit activity (spike trains) in the GP and STN of 6-OHDA-lesioned animals. The ECoG was filtered between 10 and $30 \mathrm{~Hz}$, and then the peaks of the cortical $\beta$ oscillation determined (Spike2). Phase histograms were constructed in Spike2 and then the data were exported for testing with standard circular statistics (Oriana v. 2.0; Kovach Computing Services). Modulations of single-unit activity in time with cortical $\beta$ oscillations were tested for significance using Rayleigh's Uniformity Test. The null hypothesis for Rayleigh's test was that the spike data were distributed in a uniform manner. The mean angle of spike firing, with respect to the peaks of the cortical oscillation, was also determined for each neuron. The Watson-Williams $F$ test was used to examine whether groups of neurons differed significantly in their mean angles.

Further statistical testing. The single-sample Kolmogorov-Smirnov test was used to judge whether data sets were normally distributed ( $p \leq$ 0.05 to reject). Because some data sets were not normally distributed, we used nonparametric statistical testing throughout (SigmaStat; Systat Software). The Mann-Whitney rank sum test was used for comparisons of unpaired data, whereas the Wilcoxon Signed Rank Test was used to compare paired data sets. For multiple group comparisons, we performed a Kruskal-Wallis ANOVA on ranks, with Dunn's test for further post hoc definition of comparisons. Significance for all statistical tests was set at $p<0.05$ unless noted otherwise. 


\section{Results}

Two key aims of this study were to investigate the neural basis of abnormally synchronized oscillations in the GP at the level of single neurons, small neuronal ensembles and larger neuronal populations, and to elucidate the functional significance of any disturbed activity in GP for that ongoing in STN (and vice versa). To achieve these aims, we sampled single-unit activity and LFPs from numerous sites in the GP (and STN) using linear arrays with multiple, spatially defined recording contacts (silicon probes) (Magill et al., 2006; Mallet et al., 2008). The GP and STN are located deep $(5.5-8 \mathrm{~mm})$ in the adult rat brain, which greatly challenges the use of these recording arrays in behaving rodents. We thus performed our recordings in urethane-anesthetized animals, which had the additional important benefit of allowing us to record with the probes during two well defined and controlled brain states, slow-wave activity (SWA) and cortical "activation" (Magill et al., 2006). The former state is dominated by lowfrequency rhythms $(\leq 10 \mathrm{~Hz})$ and is qualitatively similar to natural sleep, whereas the latter contains patterns of activity that are more analogous to those observed during waking behavior (Steriade, 2000). It was essential to sample activity across different brain states, as defined by electrocorticogram (ECoG) recordings, because they greatly impact upon the functional organization and coupling of rhythms within and between BG nuclei (Magill et al., 2006). Importantly, we have already established the utility of urethane-anesthetized (lesioned) rats for studying the emergence of pathological $\beta$ oscillations after dopamine loss (Mallet et al., 2008).

\section{Dopamine loss alters single-cell and network activity in GP during slow-wave activity}

We recorded the spontaneous activity of 171 GP neurons in control rats $(n=18)$ and 487 GP neurons in lesioned rats $(n=27)$ during SWA (Fig. $1 A, B$ ). In control rats, single GP neurons typically fired at high frequencies $(15-45 \mathrm{~Hz})$ and in a tonic (regular) manner (Fig. 1A), as exemplified by the presence of multiple peaks and troughs in their auto-correlograms (Fig. 1C). Analysis of activity histograms confirmed that in control rats the spike trains of most GP neurons were not modulated in time with the cortical slow $(\sim 1 \mathrm{~Hz})$ oscillation (Figs. $1 E, 2 A)$. In contrast, in lesioned rats, the spontaneous activity of GP neurons was more irregular, such that neurons exhibited rhythmic pauses or reductions in activity and thus, tended to discharge in time with the cortical slow oscillation (Fig. $1 B$ ). However, their discharges were still relatively regular within the discrete epochs of firing (Fig. $1 D)$. Importantly, two distinct types of GP neuron were identified in lesioned animals according to their firing relationships with the cortical slow oscillation (Fig. $1 F$ ). Type-A GP neurons (GP-TA) preferentially discharged during the positive "active" component of the slow $(\sim 1 \mathrm{~Hz})$ oscillation in ECoGs, which is correlated with the synchronous discharges of cortical projection neurons. In contrast, Type-I GP neurons (GP-TI) preferentially discharged during the negative "inactive" component in ECoGs, which is correlated with a widespread and profound reduction in cortical neuron activity. When recording stability allowed, we confirmed that these firing relationships were stable over several hours (data not shown). In control rats, 75\% of GP neurons had tonic activity and thus, were not modulated in time with the slow oscillation, $10 \%$ were classified as GP-TI neurons and $15 \%$ as GP-TA neurons (Fig. $2 A$ ). In contrast, in lesioned rats, most GP neurons (72\%) were classified as GP-TI, $17 \%$ as GP-TA, and only $12 \%$ as not modulated. These proportions were significantly different in control and lesioned animals ( $\chi^{2}$ analysis of contin- gency). To assess the net output activity of all GP neurons recorded in control or lesioned animals, we averaged all the activity histograms. In good agreement with observations of single cells, the net activity of GP neurons in control animals was not significantly related to a specific component of the slow oscillation (Fig. 2 B). However, in lesioned animals, the net output from the entire GP population was imbalanced, with a significantly lower firing rate during the active component, consistent with the preponderance of GP-TI neurons ( $p<0.05, \chi^{2}$ test) (Fig. $2 B$ ). This imbalance could sculpt abnormal low-frequency oscillations in STN and BG output nuclei during SWA (Magill et al., 2001; Belluscio et al., 2003; Walters et al., 2007).

Next, we assessed GP neuronal ensembles and tested for synchronized and/or oscillatory activity between pairs of spike trains ( $n=612$ and 1871 GP pairs in control and lesioned animals, respectively). Synchronization was qualitatively assessed in the time domain with cross-correlograms and quantified in the frequency domain with coherence analysis. In control rats, pairs of GP neurons were typically not correlated, as exemplified by flat cross-correlograms and coherence that was seldom significant at the dominant frequencies of SWA $(0.4-1.6 \mathrm{~Hz})$ (Fig. 1G). Only $17.6 \%$ of pairs were significantly coherent and thus, although GP neurons are powerful single-cell oscillators (Nambu and Llinaś, 1994; Stanford, 2003; Surmeier et al., 2005), these rhythms are poorly coordinated at the ensemble level when the dopaminergic system is intact. After 6-OHDA lesions, however, most pairs of GP neurons were synchronized (peak or trough at $\mathrm{t} \approx 0$ in crosscorrelograms, Fig. $1 H$ ) in an oscillatory manner (side peaks and troughs). Accordingly, $67.1 \%$ of GP unit pairs were significantly coherent at the dominant SWA frequencies $(0.4-1.6 \mathrm{~Hz})$. The average peak coherence for all GP unit pairs occurred at $\sim 1 \mathrm{~Hz}$ in both control and lesioned rats but was significantly larger in the latter (Fig. 2C). Note that GP unit pairs were only rarely coherent at $\beta$ frequencies $(15-30 \mathrm{~Hz})$ during SWA $(2.4 \%$ and $2.9 \%$ of pairs were significantly coherent in control and lesioned animals). Our simultaneous recordings of single-unit activity in GP in lesioned animals suggested that neurons of the same type tended to fire together (they were "in-phase") whereas different types of neuron tended not to do so ("anti-phase"). Our analysis of the instantaneous phase relationships between pairs of significantly coherent units supported this observation. When all types of GP neuron were analyzed together, phase differences across all unit pairs clustered at $\sim 0$ and 180 degrees (Fig. $2 D$ ). Phase differences between pairs of GP-TI neurons $(n=760)$ or between pairs of GP-TA neurons $(n=56)$ clustered at $\sim 0$ degree (Fig. $2 E)$, but the pairs of GP-TI versus GP-TA neurons $(n=328)$ clustered at $\sim 180$ degrees (Fig. 2 E).

\section{Emergence of excessive $\boldsymbol{\beta}$ oscillations in the GP depends on dopamine loss and brain state}

Exaggerated $\beta$ oscillations in the cortex and STN of anesthetized 6-OHDA-lesioned animals only occur during cortical activation (Mallet et al., 2008). We tested whether $\beta$ oscillations in GP are similarly dependent on brain state by recording population activity, as evinced in LFPs, which are highly sensitive to changes in synchronized network oscillations. The 6-OHDA lesions profoundly exaggerated $\beta$ oscillations in both cortex and GP during the activated state but not during SWA (Fig. $3 A, B$ ). The mean peak frequency in the $\beta$ band was similar in ECoGs and GP-LFPs $(20.8 \pm 0.3 \mathrm{~Hz}$ and $19.6 \pm 0.4 \mathrm{~Hz}$, respectively). The peak frequencies in the SWA band were also similar in control and lesioned animals $(0.92 \pm 0.03 \mathrm{~Hz}$ and $0.95 \pm 0.02 \mathrm{~Hz}$, respectively). The transition from SWA to cortical activation was accompanied 


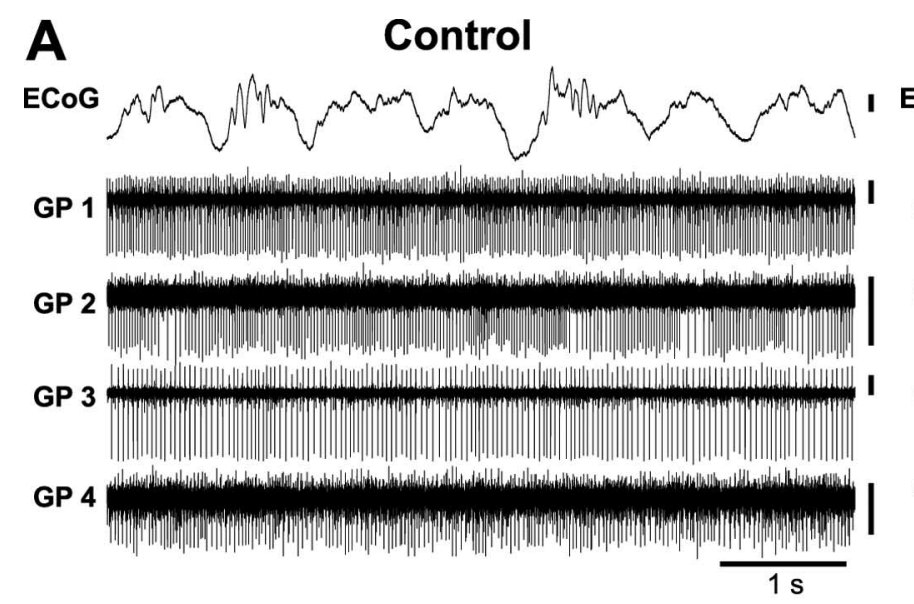

B Lesioned

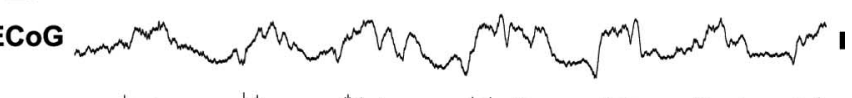

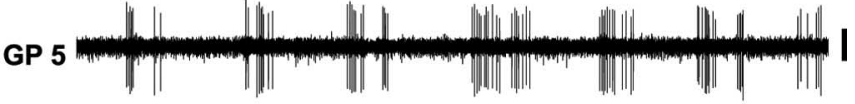

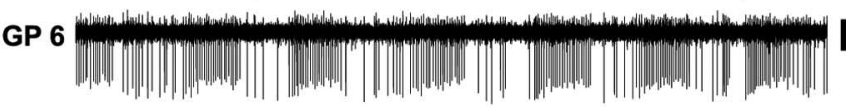

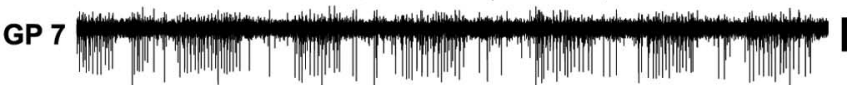
GP 8

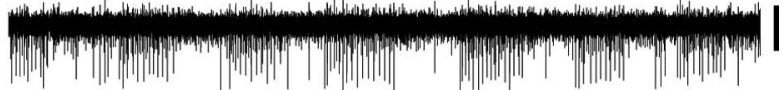

C

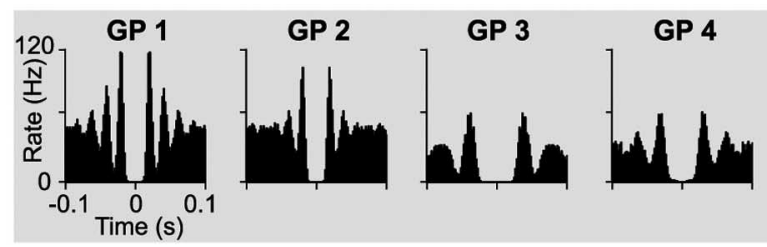

D

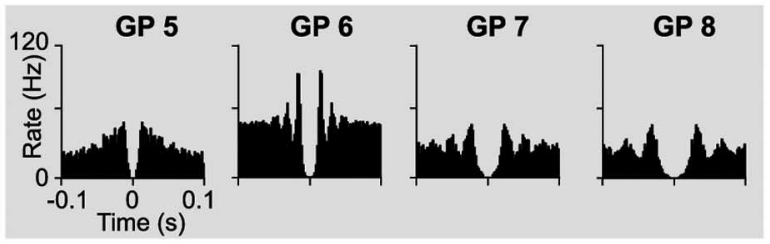

E

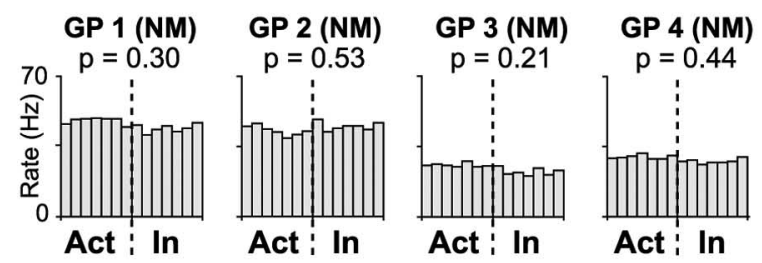

$\mathbf{F}$
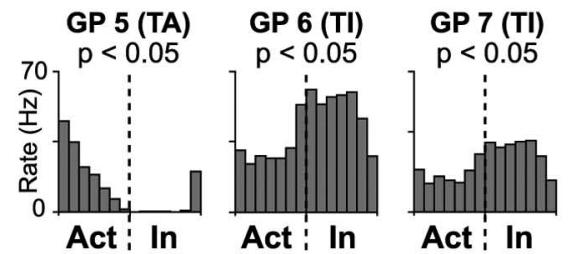

GP 8 (TI)

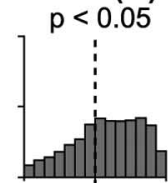

H

G GP 1

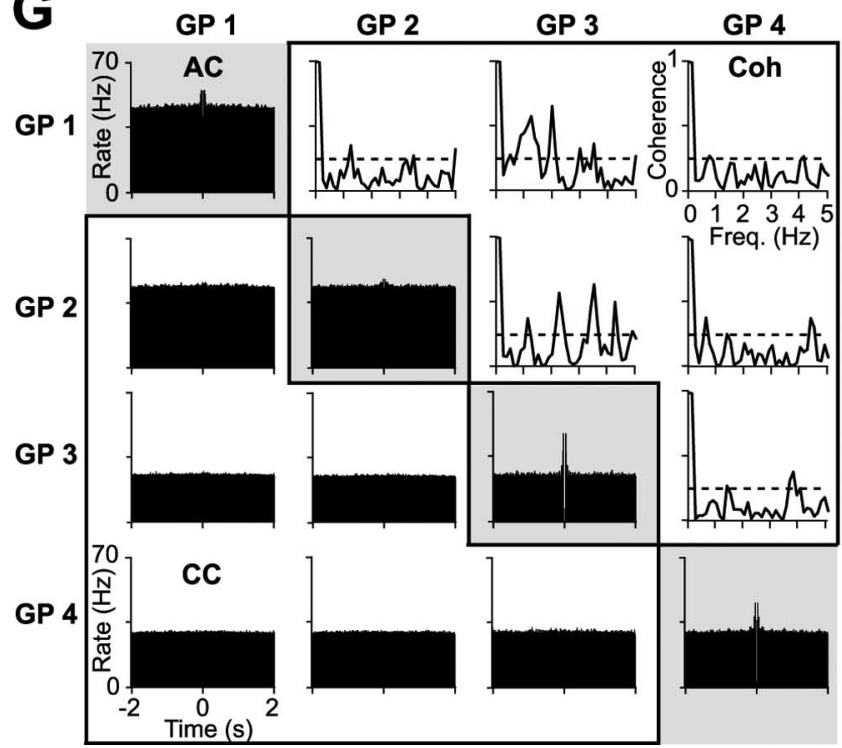
GP 3

GP 4

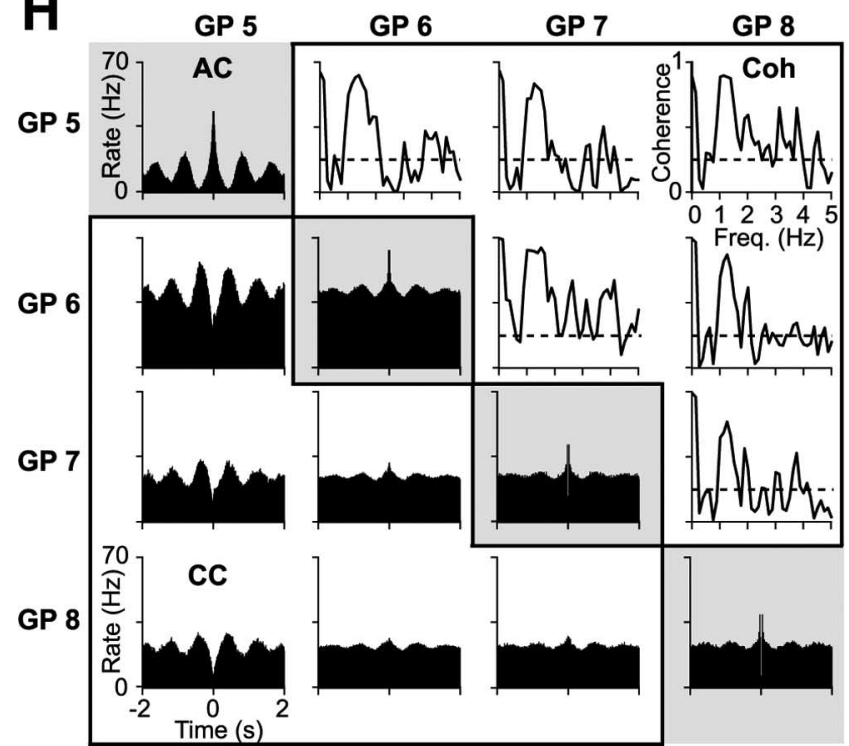

Figure 1. Single-cell and network activity in the globus pallidus of control and 6-OHDA-lesioned rats during cortical slow-wave activity. $A, B$, Simultaneous recordings of GP neurons during cortical SWA in a typical control rat ( $\boldsymbol{A}$, GP neurons $1-4)$ and a typical lesioned rat ( $\boldsymbol{B}, \mathrm{GP}$ neurons $5-8)$. Cortical activity (ECOG) is dominated by a slow oscillation at $\sim 1 \mathrm{~Hz}$. Calibration: $200 \mu \mathrm{V}$ (ECoG), $100 \mu \mathrm{V}$ (units). All subsequent panels refer to these same GP neurons. C, D, Auto-correlograms ( 2 ms bins) of GP neurons in the control ( $($ ) and lesioned rat (D). Peaks and troughs indicate the fast oscillatory nature of single-cell firing (decreased activity at time $\approx 0$ is due to the refractory period). $E$, $F$, Activity histograms of GP neurons in relation to the active (Act) or inactive (In) components of the cortical slow oscillation. In control rats, GP neurons typically fire independently of the slow oscillation components and are thus not modulated (NM) (E). Two distinct populations of GP neuron were identified in lesioned rats $(\boldsymbol{F})$. Type A neurons (TA) discharge preferentially during the active component (see GP 5) whereas type I neurons (TI) discharge preferentially during the inactive component (GP $6-8) . \mathbf{G}, \boldsymbol{H}$, In control rats (G), pairs of GP units are typically not correlated, as exemplified by flat cross-correlograms (CC, bottom left). Coherence (Coh, top right) values for pairs are typically below significance at slow oscillation frequencies ( $p=0.05$, dashed line). Long time-scale auto-correlograms ( $10 \mathrm{~ms}$ bins, $\mathrm{AC}$ ) are also flat (except att $\approx 0)$. In contrast, in lesioned rats $(\boldsymbol{H})$, pairs of GP units are typically synchronized in a slow oscillatory manner. Coherence values are significant and peak at the predominant SWA frequencies $(0.4-1.6 \mathrm{~Hz})$. Auto-correlograms (10 ms bins) indicate the slow oscillations in the spike trains of individual GP neurons. 

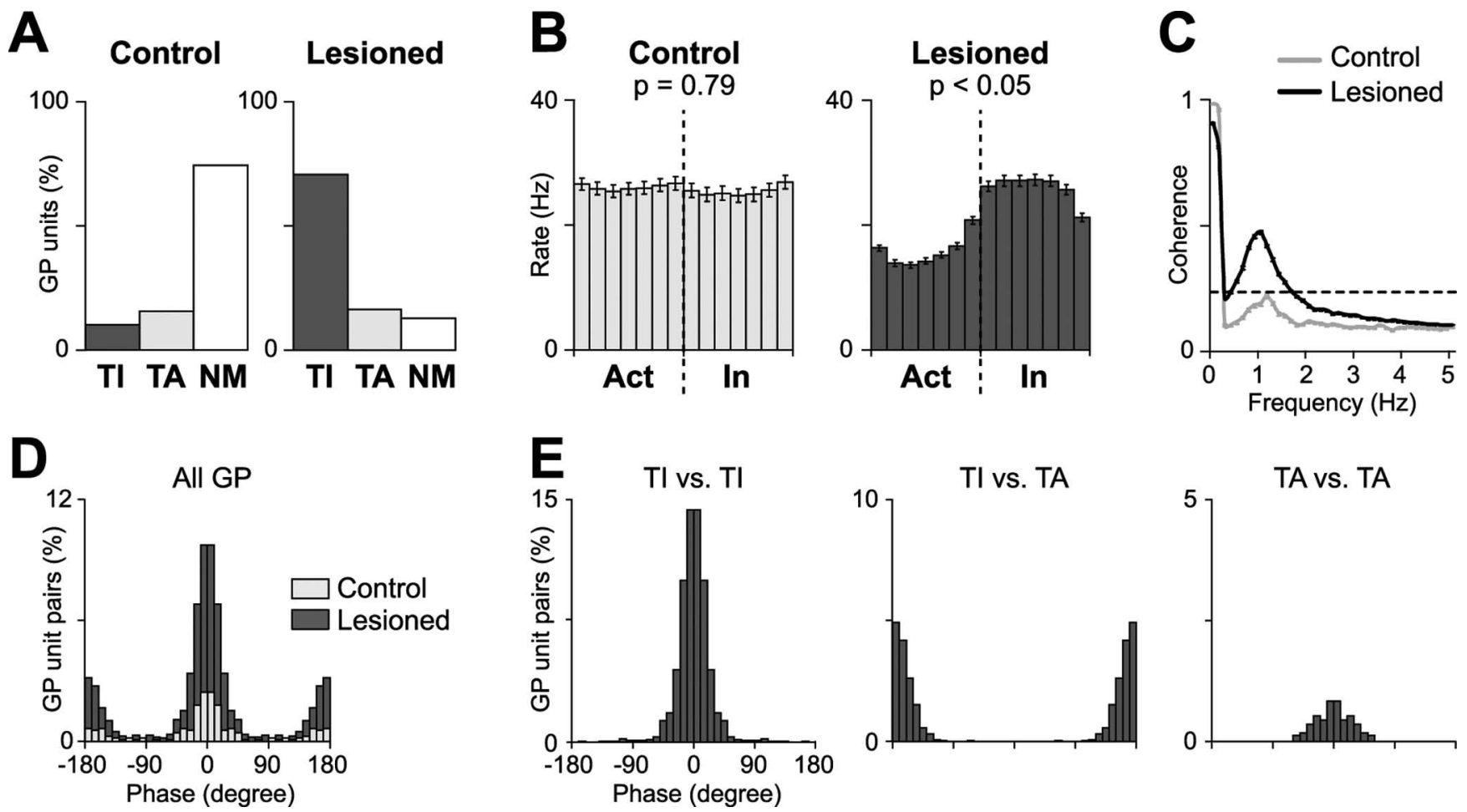

Figure 2. Population analyses of globus pallidus neuron spike rate and timing in control and 6-OHDA-lesioned rats during cortical slow-wave activity. $\boldsymbol{A}$, Proportions of the three populations of GP neuron (type I [TI], Type A [TA] and not modulated [NM]) in control and lesioned rats. $\boldsymbol{B}$, Mean activity histograms for all GP neurons in control rats (171 neurons) and lesioned rats (483 neurons) during cortical SWA. Data are means \pm 1 SEM. C, Mean coherence of all GP unit pairs tested in control rats (612 pairs) and lesioned rats (1871 pairs) during SWA. Data are means \pm 1 SEM. Dashed line is $p=0.05 . \boldsymbol{D}$, Plot of instantaneous phase values for all significantly coherent pairs of GP units in control rats (108 pairs) and lesioned rats (1255 pairs). $\boldsymbol{E}$, Instantaneous phase relationships of coherent pairs of GP units classified as type I or Type A in lesioned rats.

by significant decreases of power in the SWA frequency band $(0.4-1.6 \mathrm{~Hz})$ for control and lesioned rats (decreased by $89.4 \pm$ $2.3 \%$ and $94.6 \pm 0.9 \%$, respectively) (Fig. $3 C$ ). The proportional decreases were similar for lesioned and control rats. However, the total $\beta$ band power $(15-30 \mathrm{~Hz})$ of ECoGs and GP-LFPs significantly increased in lesioned rats during cortical activation only (Fig. 3D).

\section{Neural basis of excessive $\boldsymbol{\beta}$ oscillations in the GP after dopamine loss}

Having demonstrated that $\beta$ oscillations were exaggerated in GPLFPs during cortical activation in lesioned rats, we next defined in more detail the neural substrates of this abnormal population activity. Local field potentials are somewhat ambiguous reflections of the underlying neuronal activity, and often better reflect synchronized subthreshold postsynaptic potentials (i.e., correlated input activity) than suprathreshold output activity (Mitzdorf, 1985; Boraud et al., 2005). Therefore, to test for the expression of $\beta$ oscillations at the level of GP outputs, we recorded the spontaneous discharges of 149 GP neurons in control rats and 478 neurons in lesioned rats during cortical activation. Cortical activation was confirmed by quantifying the frequency content of ECoG and GP-LFP power spectra (see above). Thus, activation was associated with a loss of low-frequency rhythms $(<10 \mathrm{~Hz})$, and, in lesioned rats only, the emergence of prominent $\beta$ oscillations at $\sim 20 \mathrm{~Hz}$ (Fig. $4 A, B$ ). In control rats during activation, the firing pattern of single GP units was regular and similar to that in SWA [coefficients of variation (CVs): $0.43 \pm 0.01$ and $0.49 \pm 0.01$, respectively] (Figs. $1 A, 4 A, 5 B$ ). However, GP neurons fired at a significantly higher rate during activation compared with SWA $(33.7 \pm 1.3 \mathrm{~Hz}$ and $25.9 \pm 1.0 \mathrm{~Hz}$, respectively, $p<0.001$ ) (Fig. 5A). In lesioned rats during activation, GP neurons did not display the slow $(\sim 1 \mathrm{~Hz})$ modulations in discharge that were evident during SWA but instead fired single spikes or spike "doublets" in a more regular manner than in SWA (CVs: $0.74 \pm 0.01$ and $1.28 \pm 0.02$ during activation and SWA, respectively, $p<0.001$ ) (Figs. $1 B, 4 B, 5 B$ ). This increase in firing regularity was associated with a significant decrease in firing rate compared with SWA $(14.6 \pm 0.4 \mathrm{~Hz}$ and $20.2 \pm 0.5 \mathrm{~Hz}$, respectively, $p<0.001$ ) (Fig. 5A). As compared with controls, the mean firing rate of GP neurons was significantly lower and the firing patterns were significantly less regular after lesions, regardless of brain state (Fig. $5 A, B$ ).

Next, we assessed GP neuronal ensembles and tested for synchronized and/or oscillatory activity between pairs of spike trains, as we did for SWA. In control rats, the discharges of GP unit pairs were typically not correlated in either the time or frequency domains (Fig. 4C). This suggests that oscillation and synchronization are largely distinct functional properties in the GP neuronal network when the dopaminergic system is intact, and that the intrinsic membrane properties of GP neurons, which endow autonomous pacemaking (Nambu and Llinaś, 1994; Stanford, 2003; Surmeier et al., 2005), likely dominate and underlie their fast and regular spontaneous activity rather than their inputs. In contrast, after 6-OHDA lesions, inputs to GP neurons likely had a greater impact on unit activity, such that independent autonomous pacemaking no longer dominated firing, because oscillations at the single-cell level were also expressed at the network level. Indeed, $\beta$ oscillations were often evident in auto- and cross-correlograms (Fig. 4D). This tendency for GP neurons to oscillate together at $\beta$ frequencies was reflected by significant peaks of coherence at $\sim 20 \mathrm{~Hz}$ (Fig. $4 D$ ). Accordingly, the mean 
A Slow-Wave Activity
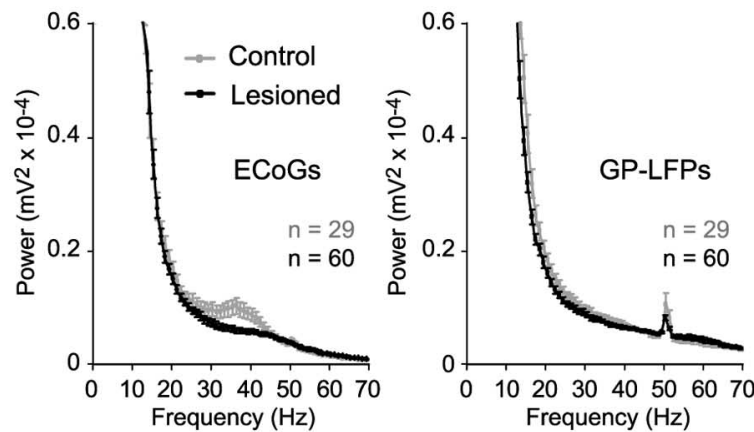

B

Activation
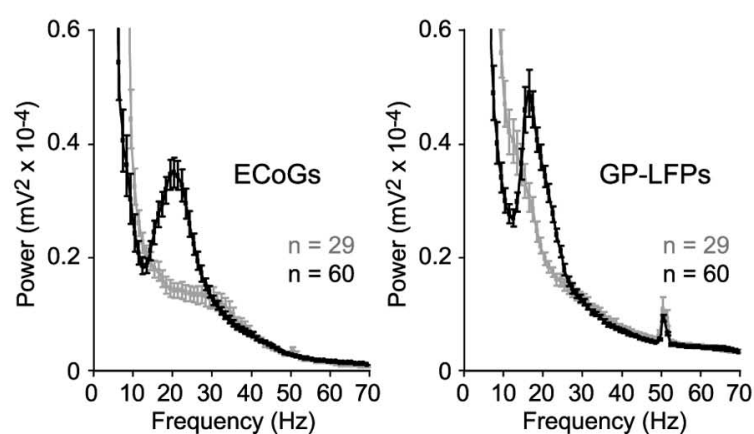

Figure 3. Brain state-dependency of the power and frequency of local field potentials in cortex and globus pallidus in control and 6-OHDA-lesioned rats. $A, B$, Average power spectra of simultaneously recorded local field potentials in cortex (ECOG) and GP (GP-LFPs) in control and lesioned rats during SWA ( $\boldsymbol{A}$ ) and cortical activation (B). During SWA, ECOG and GP-LFPs power spectra are similar in control and lesioned rats. Prominent $\beta$ oscillations emerge in cortex and GP during activated brain state in lesioned animals only. Weak power at $50 \mathrm{~Hz}$ in GP-LFP power spectra is line noise. C, Quantitative comparison of ECoG power in the SWA frequency band $(0.4-1.6 \mathrm{~Hz})$. Asterisks denote $p<0.01$. D, Quantitative comparison of ECOG power and GP-LFP power in the $\beta$ band $(15-30 \mathrm{~Hz})$ during activation. Cortical activation is accompanied by a significant decrease in SWA band power in control and lesioned rats, and a significant increase in $\beta$-band power in lesioned rats only. Data in $\boldsymbol{A}-\boldsymbol{D}$ are means \pm 1 SEM. Asterisks denote $p<0.01$ compared with the three other groups.

coherence of all GP pairs recorded in lesioned rats (2028 pairs) showed a clear and significant peak at $\beta$ frequencies (centered at $\sim 20 \mathrm{~Hz}$ ) but this was not the case in controls (491 pairs) (Fig. $5 C)$. Moreover, $44.2 \%$ of GP pairs were coherent at $\beta$ frequencies $(15-30 \mathrm{~Hz})$ after $6-O H D A$ lesion compared with $0.6 \%$ of pairs in controls. Thus, the proportion of coherent GP neuron pairs in the $\beta$-frequency band was augmented almost 100 -fold after lesions. The few pairs of GP units that were coherent in controls did not exhibit a common peak at $\sim 20 \mathrm{~Hz}$ unlike in lesioned animals (Fig. 5D). Note that, in agreement with our findings of significantly decreased power in the SWA frequency band during cortical activation, GP unit pairs were only rarely coherent at SWA frequencies $(0.4-1.6 \mathrm{~Hz})$ during cortical activation $(3.6 \%$ and $2.3 \%$ of pairs were significantly coherent in control and lesioned animals). One of the principal benefits of recording network activity with silicon probes is that the spatial arrangements of recording contacts and thus, the neurons recorded thereon, are precisely known. We took advantage of this to test how synchronized GP ensemble activity varied in space. We found that GP neuron pairs separated by as much as $1400 \mu \mathrm{m}$ could exhibit significantly coherent activity in the $\beta$-frequency range (Fig. $5 E, F)$. Given that the adult rat external globus pallidus has a volume of $\sim 2.6 \mathrm{~mm}^{3}$, and is $<2.5 \mathrm{~mm}$ in its longest axis (Oorschot, 1996), these data suggest that the inappropriate synchronization of GP neuron activity at $\beta$ frequencies spans much of this nucleus. However, both peak coherence of pairs, and the proportion of pairs that were coherent, decreased as the separation distance increased (Fig. $5 E, F$ ).

Putative monosynaptic connections between GP neuron pairs were assessed during cortical activation by testing for short-latency temporal interactions in cross-correlograms, as previously $\mathrm{de}$ scribed for cortical recordings (Barthó et al., 2004). Of the 2028 and 491 crosscorrelograms tested in the lesioned and control rats, respectively, only $3(0.2 \%)$ and $3(0.6 \%)$ GP pairs displayed significant short-latency interactions, all of which suggested nonreciprocal inhibition.

\section{Dichotomy in GP activity during SWA is} maintained during $\boldsymbol{\beta}$ oscillations

In lesioned rats, the increased rhythmic activity in GP during SWA revealed two types of neuron that fired in time with the cortical slow oscillation. Despite the fact that most GP neurons were synchronized in a slow oscillation at $\sim 1 \mathrm{~Hz}$, large phase differences were seen across the network (Figs. 1, 2). Thus, while pairs of GP-TI neurons, or pairs of GP-TA neurons, tended to synchronize with small phase differences (in-phase), pairs made up of a GP-TI neuron and a GP-TA neuron fired in anti-phase. We next addressed the important issue of whether these distinct phase relationships are maintained across the two brain states, or whether different types of GP neuron are reorganized and embedded into a single ensemble firing inphase during $\beta$ oscillations. To differentiate between these two possibilities, we recorded the GP-TI and GP-TA neurons, as identified during SWA, in a subsequent activated brain state dominated by $\beta$ oscillations (Fig. $6 A, B$ ). Time domain analysis suggested that the coupling of activity within GP pairs during SWA is maintained during $\beta$ oscillations, i.e., crosscorrelograms of TI versus TI neurons displayed a peak around $\mathrm{t} \approx 0$ in SWA and $\beta$ states, and cross-correlograms of TA versus TI neurons displayed troughs around $\mathrm{t} \approx 0$ in both brain states (Fig. 6C,D). When recording stability allowed, we confirmed that these firing relationships were stable over several hours (data not shown). Are these patterns of temporal coupling maintained across the entire GP population? To answer this key question, we performed coherence and phase analyses for all GP pairs (regardless of type), all TI versus TI pairs, all TI versus TA pairs, and all TA versus TA pairs during SWA $(n=779,526,218$, and 35 pairs, respectively) (Fig. $6 E$ ) and during $\beta$ oscillations ( $n=483,344$, 125 , and 14 pairs, respectively) (Fig. $6 F$ ) recorded in the same animals. The mean coherence and peak frequencies were similar for the different groups of GP pairs during SWA (Fig. $6 E$ ). The same was also true during exaggerated $\beta$ oscillations, with the exception that TA versus TA pairs were, on average, significantly less coherent in the 15-30 Hz range compared with the other pairings (Fig. 6 F). Importantly, the tendency for instantaneous phase differences of GP pairs to cluster around 0 or 180 degrees that occurred during SWA was also apparent during exaggerated $\beta$ oscillations (Fig. $6 G, H$ ). Note the increased spread of phase 
A

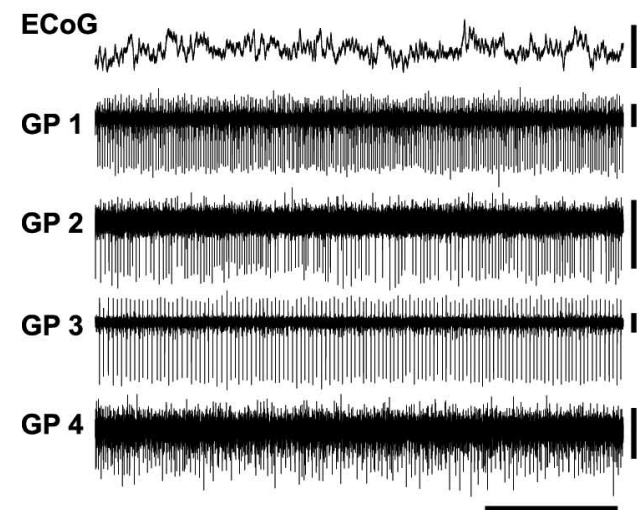

$1 \mathrm{~s}$

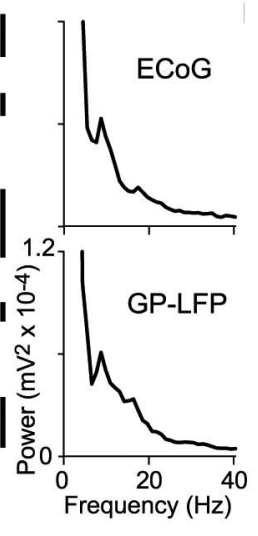

B

\section{Lesioned}

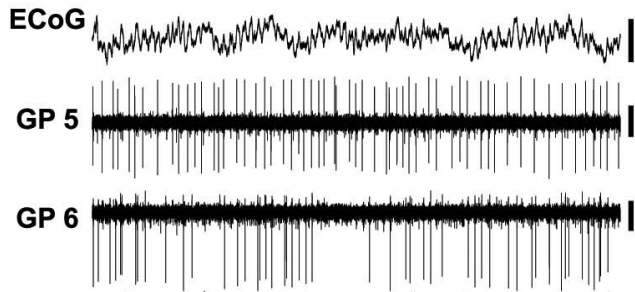

GP 7
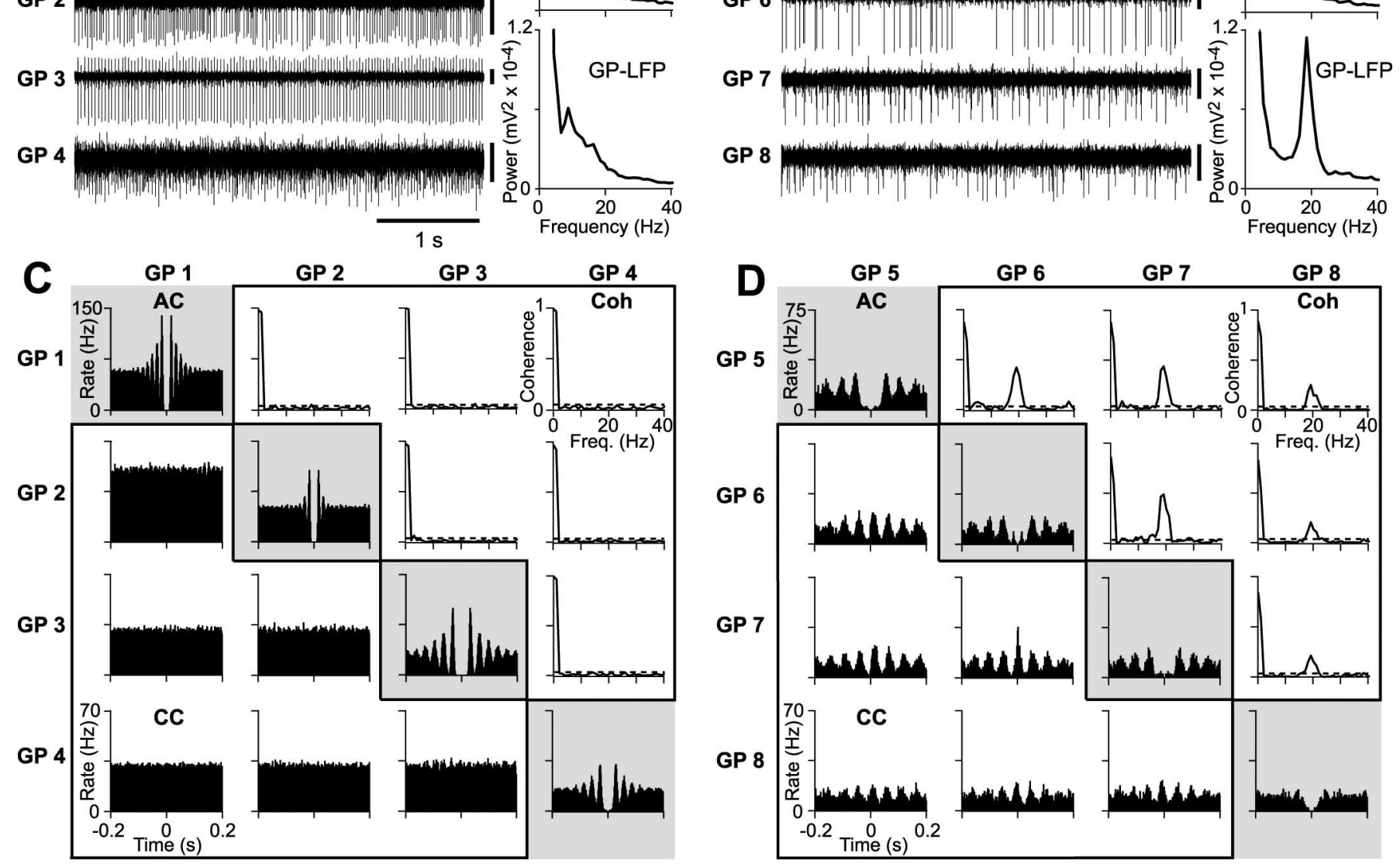

Figure 4. Single-cell and network activity in the globus pallidus of control and 6-OHDA-lesioned rats during cortical activation. $A$, $B$, Same GP neurons as in Figure 1 during subsequent epochs of activated brain states. Power spectra of field potentials in cortex (ECOG) and globus pallidus (GP-LFPs) show the excessive $\beta$ oscillations ( $\sim 20 \mathrm{~Hz}$ ) in the lesioned rat compared with the control. Calibration: $200 \mu \mathrm{V}(\mathrm{ECOG}), 100 \mu \mathrm{V}$ (units). C, D, During activated state in control rats (C) pairs of GP units are typically not correlated (flat cross-correlograms; CC). Peaks and troughs in auto-correlograms (AC, 2 ms bins) indicate the fast oscillatory nature of single-cell firing. Coherence (Coh) values for pairs were typically below significance ( $p=0.05$, dashed line). In contrast, in lesioned rats $(\boldsymbol{D})$, pairs of GP neurons tend to synchronize and coherence values peak at $\beta$ frequencies $(\sim 20 \mathrm{~Hz})$.

differences in the phase plots of activity during $\beta$ oscillations does not mean that activity is less tightly organized in time compared with that during SWA because a single phase bin (10 degrees) during $\beta$ represents $1.4 \mathrm{~ms}$ (assuming a cycle period of $50 \mathrm{~ms}$ ) whereas a phase bin during SWA is $28 \mathrm{~ms}$ (assuming a cycle period of $1000 \mathrm{~ms}$ ). This dichotomy in the temporal coupling of different types of GP neuron was also reflected in their different firing rates and patterns (Fig. 6I,J). Thus, GP-TI and GP-TA neurons have significantly different firing rates in SWA $(24.2 \pm$ $0.7 \mathrm{~Hz}$ and $11.8 \pm 1.1 \mathrm{~Hz}$, respectively) and during $\beta$ oscillations $(14.1 \pm 0.5 \mathrm{~Hz}$ and $19.4 \pm 1.4 \mathrm{~Hz}$, respectively). However, these differences were dependent on brain state, such that GP-TI neurons fired faster than GP-TA neurons during SWA but slower than GP-TA neurons during $\beta$ oscillations (Fig. $6 I, J$ ). Similarly, the firing regularity of GP-TI and GP-TA neurons was inversely related but varied according to brain state. Indeed, GP-TI neurons were more regular than GP-TA neurons during SWA but were less regular than GP-TA neurons during $\beta$ oscillations (Fig. $6 I, J)$. Thus, the dichotomy in the temporal couplings of different types of GP neuron is maintained across two extreme network oscillations, SWA and $\beta$. Together with the dichotomy of GP firing rates and patterns at the single-cell level, this underlies a complexity that cannot be easily appreciated when GP neurons are considered as a single population.

\section{Orchestration of abnormal oscillations in the STN-GP} network after dopamine loss

The subthalamic nucleus (STN) is reciprocally connected with the GP. The STN provides the main excitatory glutamatergic input to GP, whereas the GP provides the main inhibitory GABAergic input to STN (Smith et al., 1998). To understand better how the abnormal oscillations observed in lesioned rats were generated, we conducted simultaneous recordings of STN and GP neurons in control and lesioned rats (6 and 11 rats, respectively). Single units and LFPs were recorded in STN with silicon probes or glass electrodes (data pooled together) in control and lesioned rats during SWA (23 and 32 neurons, respectively) and/or cortical activation (23 and 49 neurons, respectively). During SWA in 

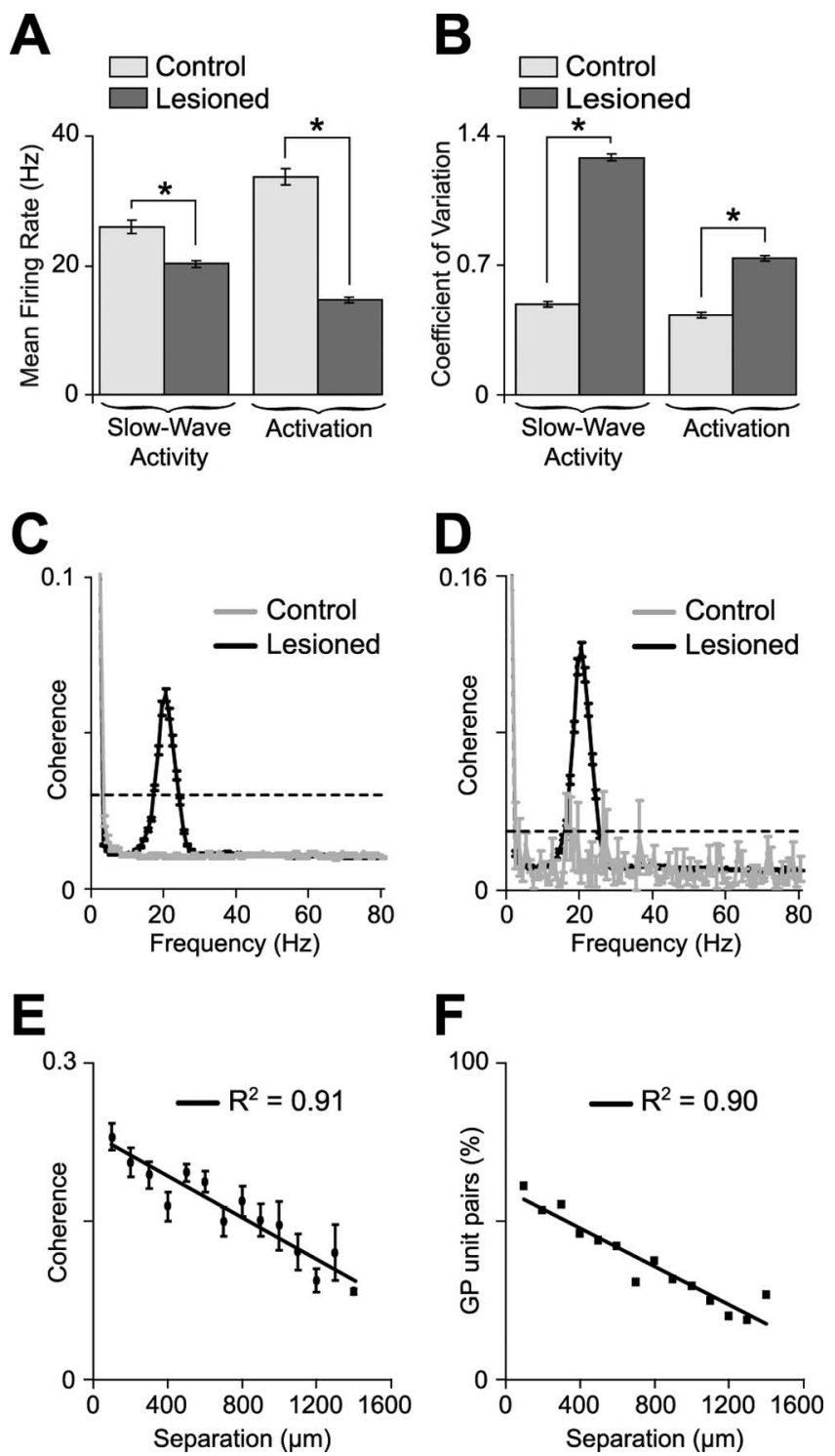

Figure 5. Excessive synchronization of globus pallidus ensemble activity after 6-0HDA lesion is associated with decreases in the firing rate and regularity of single neurons. $A, B$, Average firing rates and coefficients of variation of all GP neurons during SWA and cortical activation in control rats (171 and 149 neurons, respectively) and lesioned rats (487 and 478 neurons, respectively). Asterisks denote $p<0.01$. C, Mean coherence of all GP unit pairs tested in control rats (491 pairs) and lesioned rats (2028 pairs) during cortical activation. Note appearance of significant coherence peak at $\beta$ frequencies during activation in lesioned rats. $\boldsymbol{D}$, Mean coherence of all GP unit pairs with significant coherence in the $\beta$-frequency band $(15-30 \mathrm{~Hz})$ in control rats ( 3 pairs) and lesioned rats ( 897 pairs) during cortical activation. Dashed line in $C$ and $\boldsymbol{D}$ is $p=0.05 . \boldsymbol{E}, \boldsymbol{F}$, Correlation between the separation of significantly coherent GP unit pairs and peak coherence of pairs in the $\beta$ band $(\boldsymbol{E})$, and between separation and the percentage of significantly coherent GP pairs $(\boldsymbol{F})$, in lesioned animals. Data in $\boldsymbol{A}-\boldsymbol{E}$ are means \pm 1 SEM.

either control or lesioned rats, STN neurons preferentially discharged during the active component of the cortical slow oscillation (supplemental Fig. $1 A, B, E$, available at www.jneurosci.org as supplemental material). In agreement with our recent findings (Mallet et al., 2008), excessive $\beta$ oscillations were expressed in STN unit activity and STN-LFPs during cortical activation in lesioned animals only (supplemental Fig. $1 C, D, F$, available at www.jneurosci.org as supplemental material). We found that STN neurons were hyperactive during both brain states in lesioned rats compared with controls (supplemental Fig. 1G, available at www.jneurosci.org as supplemental material). The coeffi- cients of variation of STN activity decreased in lesioned rats compared with controls during activation but not SWA (supplemental Fig. $1 G$, available at www.jneurosci.org as supplemental material). Thus, increased oscillatory activities at $\beta$ frequencies in the STN of lesioned rats are associated with increases in both the mean rate and regularity of firing.

We analyzed the spike timings of STN and GP neurons simultaneously recorded in lesioned rats $(n=11)$ during $\beta$ oscillations. All GP neurons were identified as either GP-TI or GP-TA neurons during a previous SWA epoch. During $\beta$ oscillations, STN neurons discharged a "burst" of spikes or a single spike in time with most (but not necessarily all) $\beta$ cycles (supplemental Fig. $1 D$, available at www.jneurosci.org as supplemental material) (Fig. 7A). Neither type of GP neuron fired bursts of spikes, but instead fired a single spike, or sometimes a spike doublet, during most $\beta$ cycles (Figs. 6B, 7A). Cross-correlograms of STN-GP neuron pairs showed a synchronization of oscillatory activity at $\beta$ frequencies but the temporal coupling depended on the type of GP neuron recorded (Fig. 7B). Thus, STN versus GP-TA pairs tended to fire in-phase (peak at time $\approx 0$ ) whereas STN versus GP-TI pairs tended to fire in anti-phase (trough at time $\approx 0$ ) (Fig. $7 B)$. Significant short-latency interactions between STN-GP pairs suggestive of monosynaptic connections were not observed. To characterize precisely the phase relationships between STN, GP-TA, and GP-TI neurons, we calculated linear phase histograms for each type of neuron $(39,41$, and 59 neurons, respectively) while using the peaks of the coincident cortical $\beta$ oscillation as a common reference signal. The mean peak frequency of the cortical $\beta$ oscillations in these rats was $20.5 \pm 0.4 \mathrm{~Hz}$. The probabilities that STN and GP neurons fired varied according to the phase of the ongoing $\beta$ oscillations (Fig. 7C). On average, STN neurons were most likely to fire at $244 \pm 6$ degrees with respect to the peak of the cortical oscillation (Fig. 7C). GP-TA neurons fired at $266 \pm 6$ degrees whereas GP-TI neurons fired at $37 \pm 7$ degrees (Fig. 7C). These differences in the mean phase angles of firing were significant $(p<0.02$, Watson-Williams $F$ test). Thus, during each $\beta$ cycle, the peak activity of GP-TI neurons coincided with minimal activity of STN and GP-TA neurons, while peak activities of STN and GP-TA neurons coincided with minimal activity of GP-TI neurons. Note that although the phase of the ECoG $\beta$ oscillations at which cortical projection neurons are most active is unknown, the cortical oscillations still serve as a useful common reference for examining the phase differences between neurons in the STN-GP network. Indeed, the phase relationships between STN and GP neurons that we have defined would still stand regardless of whether ECoG peaks or troughs were used as a reference. Our results from analysis of the linear phase histograms were supported by frequency domain analyses. Coherence was calculated for STN versus STN pairs, STN versus all GP pairs, STN versus GP-TI pairs and STN versus GP-TA pairs (7, 156, 132 and 24 pairs, respectively). Significant coherence peaked at $\sim 20 \mathrm{~Hz}$ for all pairings tested (Fig. 7D). The instantaneous phase differences for STN versus STN pairs, and STN versus GP-TA pairs, clustered around 0 degree, whereas phase differences for STN versus GP-TI pairs clustered around 180 degrees (Fig. 7E). Therefore, during excessive $\beta$ oscillations, neurons in the STN-GP network synchronize and oscillate together at $\beta$ frequencies, but the spike timings suggest a complex dynamic where STN neuron discharges are in-phase with those of GP-TA neurons but are anti-phase with those of GP-TI neurons. 
A

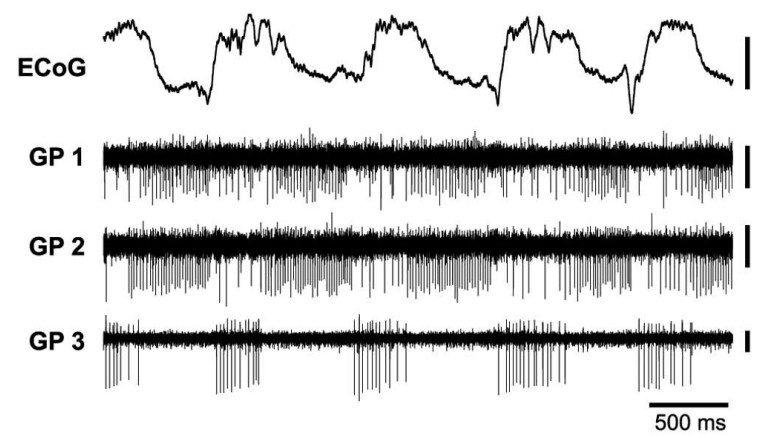

C

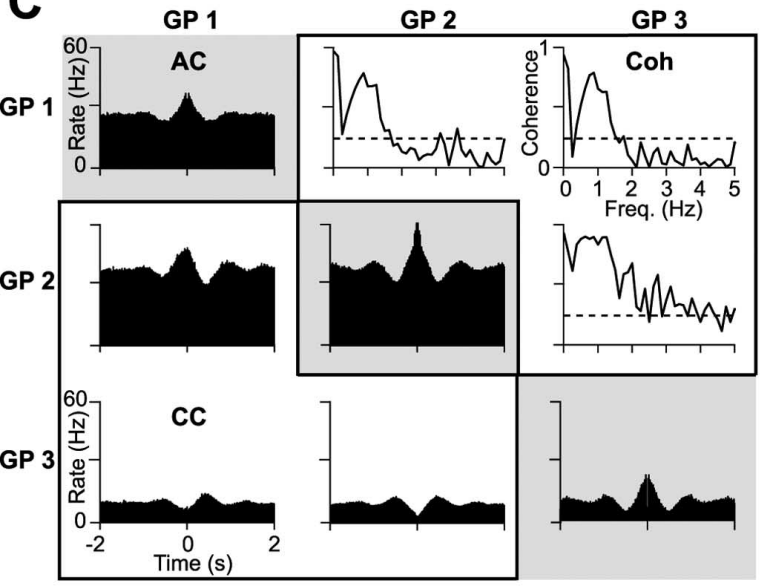

E

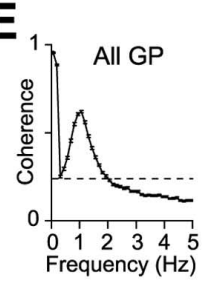

SWA
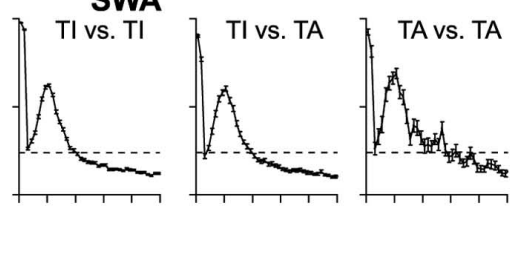

SWA
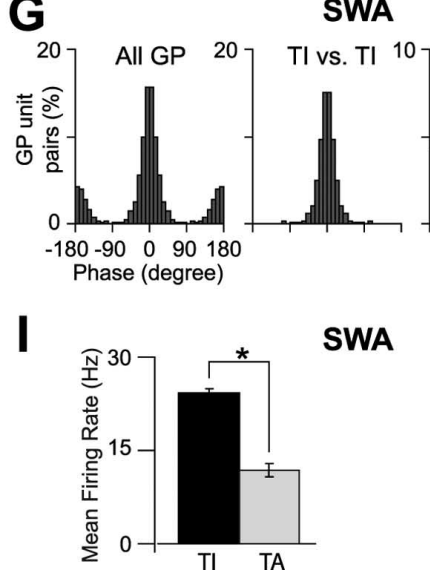

SWA

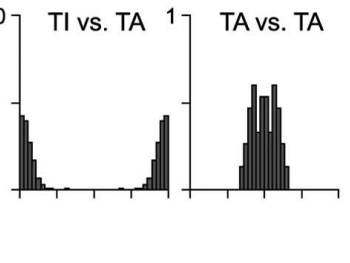

B

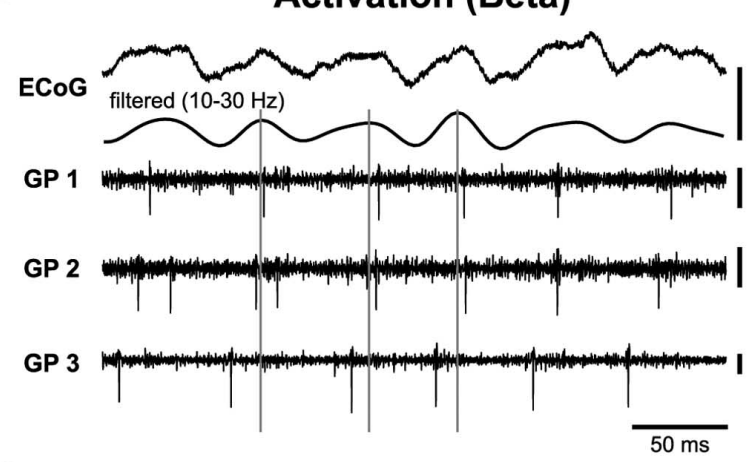

D

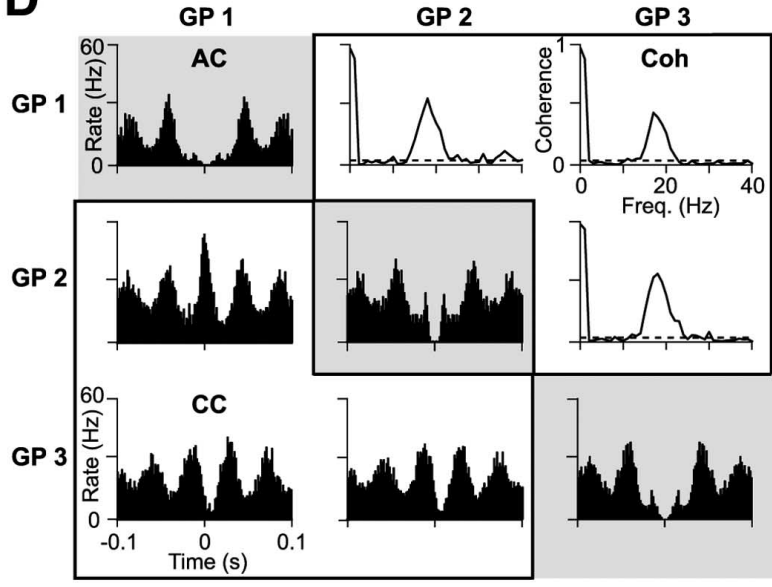

$\mathbf{F}_{0}$

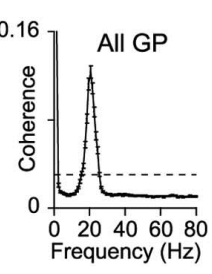

Beta
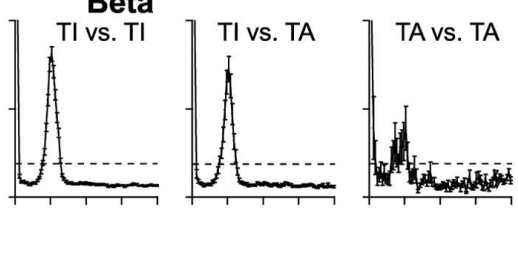

H

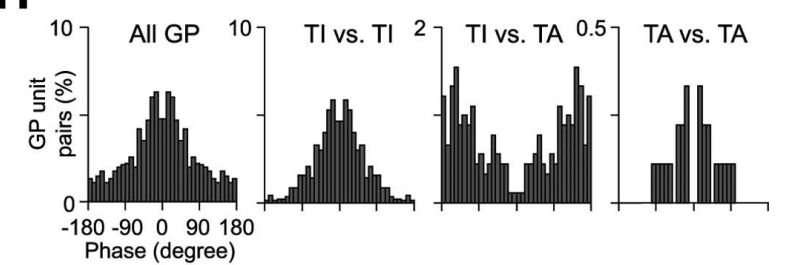

J

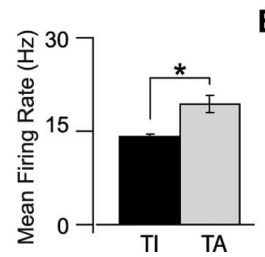

Beta

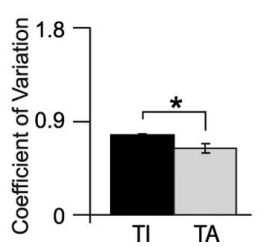

Figure 6. Dichotomy of globus pallidus unit activity in 6-0HDA-lesioned rats is maintained across slow $(\sim 1 \mathrm{~Hz})$ and $\beta(\sim 20 \mathrm{~Hz})$ network oscillations. $A$, $\boldsymbol{B}$, Simultaneous recordings of three GP neurons [GP 1 and GP 2 are type I (TI), and neuron GP 3 is Type A (TA)] in a lesioned rat during SWA ( $\boldsymbol{A}$ ) and a subsequent activated state dominated by $\beta$ oscillations $(\boldsymbol{B})$. Gray lines centered on peaks of the cortical $\beta$ oscillation exemplify spike timing relationships. Calibration: $500 \mu \mathrm{V}$ (ECOG,SWA), $200 \mu \mathrm{V}$ (ECoG, $\beta$ ), and $100 \mu \mathrm{V}$ (units). C, D, Auto-correlograms (AC), cross-correlograms (CC) and coherence plots (Coh) for the same GP neurons demonstrating the dependency of synchronization and the frequency of oscillatory activity on brain state. $10 \mathrm{~ms}$ bins for $\mathrm{AC}$ and $\mathrm{CC}$ in $\mathrm{C} ; 2 \mathrm{~ms}$ bins in D. Note that temporal relationships established between types of GP unit during SWA are maintained during $\beta$ oscillations. $E, F$, Average coherence plots for all significantly coherent GP unit pairs during SWA $(\boldsymbol{E})$ or during $\beta$ oscillations prevalent in the activated brain state $(\boldsymbol{F})$ in all lesioned rats. Note significant peaks of coherence for all GP pairs, all TI versus TI pairs, all TI versus TA pairs, and all TA versus TA pairs were at $\sim 1 \mathrm{~Hz}$ during SWA $(\boldsymbol{E})$ and were at $\sim 20 \mathrm{~Hz}$ during activated state $(\boldsymbol{F}) . \boldsymbol{G}, \boldsymbol{H}$, Plots of instantaneous phase values for all pairs of $G P$ neurons (same pairs as in $\boldsymbol{E}$ and $\boldsymbol{F}$ ) during SWA $(\boldsymbol{G})$ and $\beta$ oscillations $(\boldsymbol{H})$. Given mean peak frequencies of $\sim 1 \mathrm{~Hz}$ and $\sim 20 \mathrm{~Hz}$, the phase bins of 10 degrees in $(\boldsymbol{G})$ and $(\boldsymbol{H})$ represent $\sim 28 \mathrm{~ms}$ and $1.4 \mathrm{~ms}$, respectively. The clustering of phase relationships between types of GP neuron is maintained across the two extreme brain states. I,, , Average firing rates and coefficients of variation for each type of GP neuron (TI, 280 neurons; TA, 62 neurons) during SWA $(\boldsymbol{I})$ and $\beta$ oscillations $(\boldsymbol{J})$. Asterisks denote $p<0.01$. Data in $\boldsymbol{E}, \boldsymbol{F}, \boldsymbol{I}$, and $\boldsymbol{J}$ are means \pm 1 SEM. 


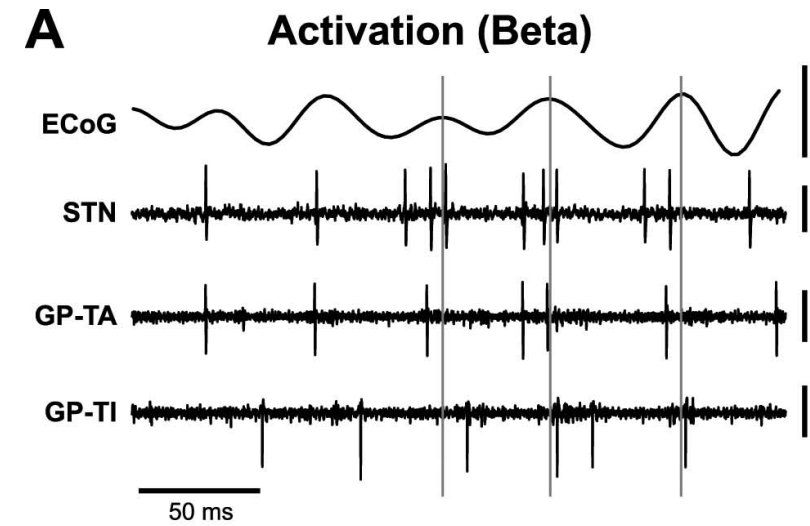

B
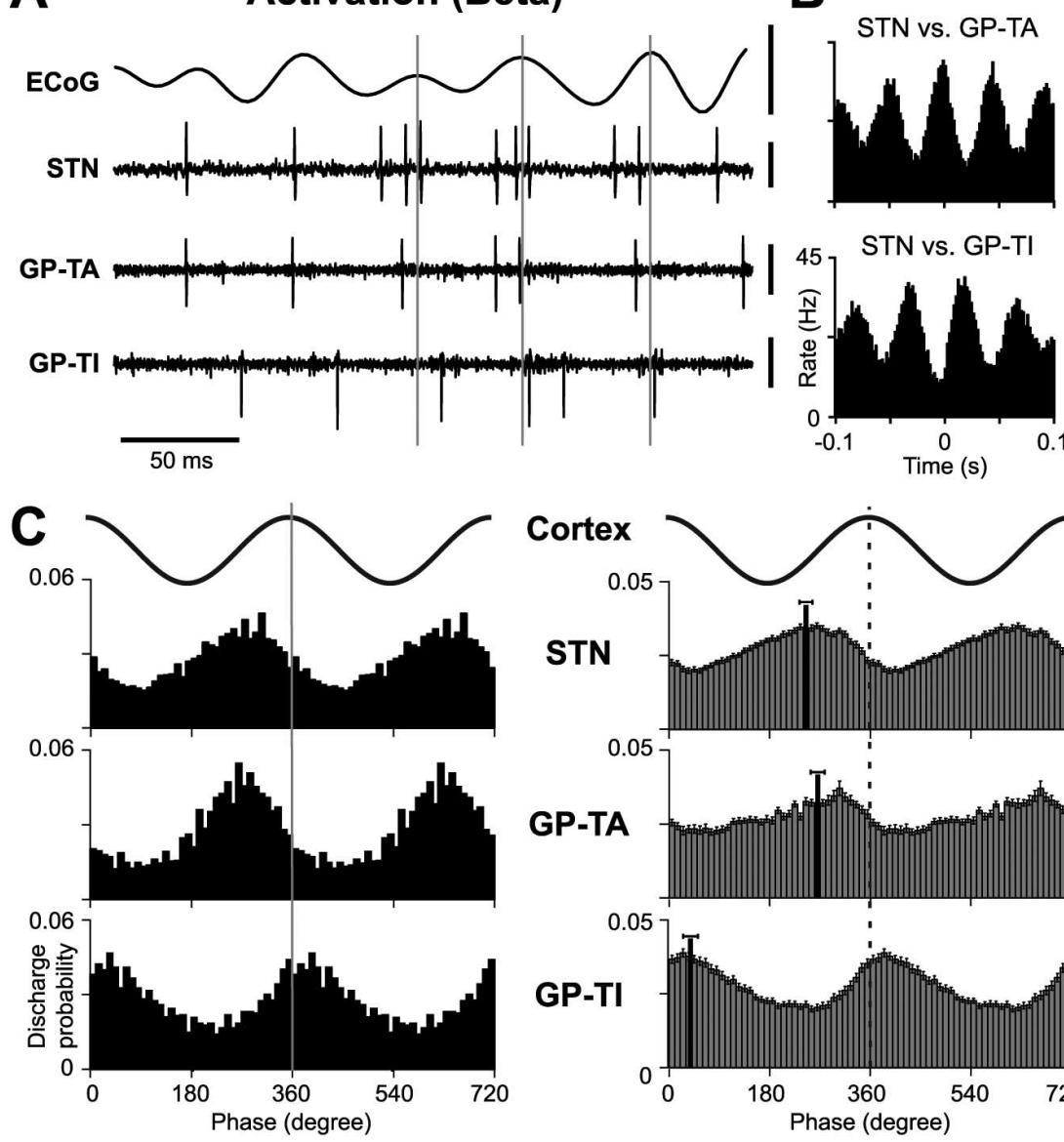

Cortex
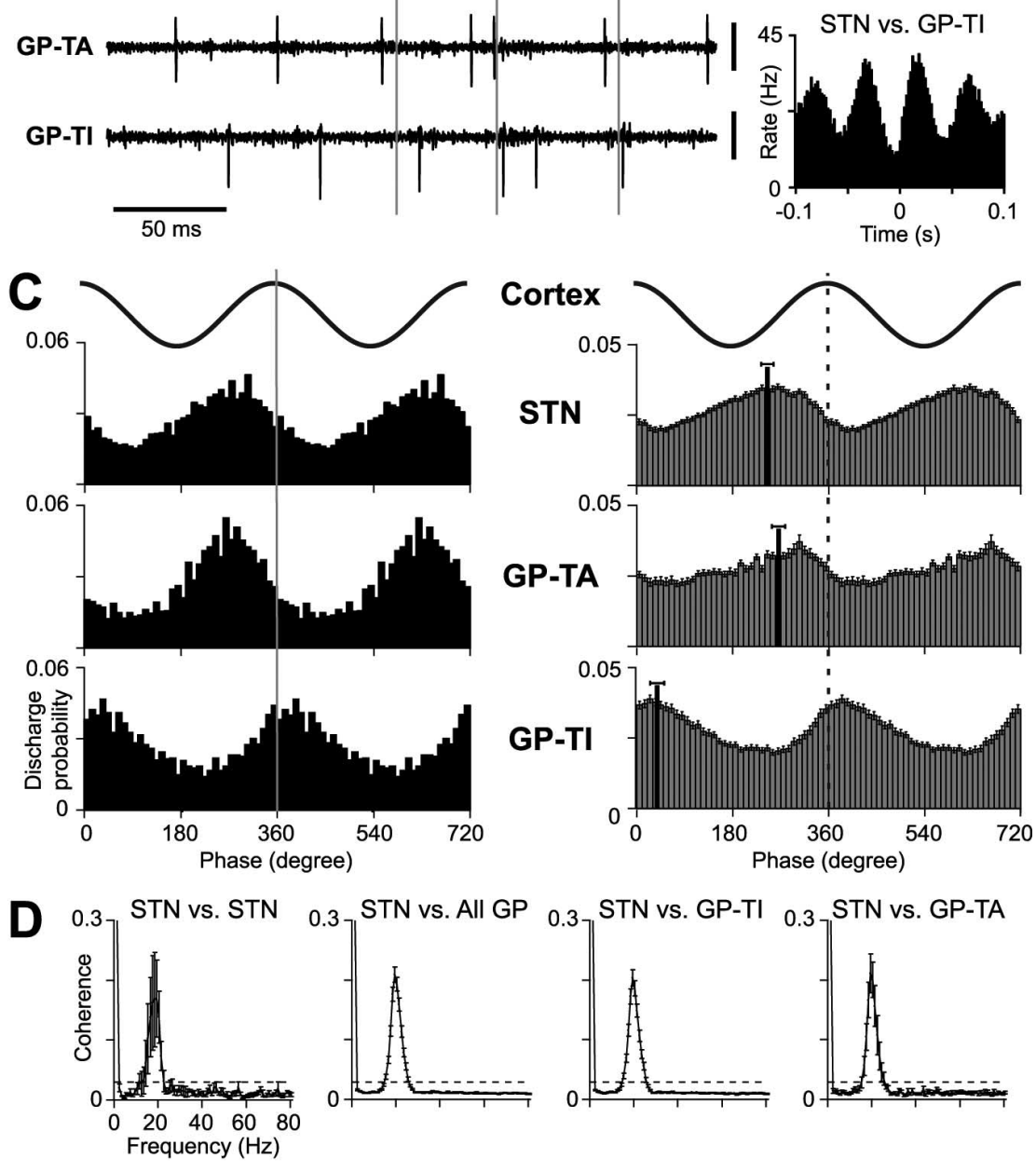

GP-TA

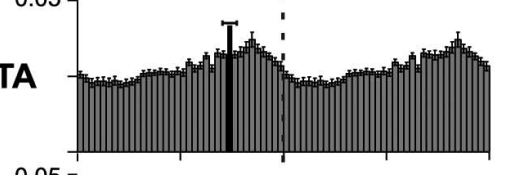

GP-TI

$$
0.05
$$
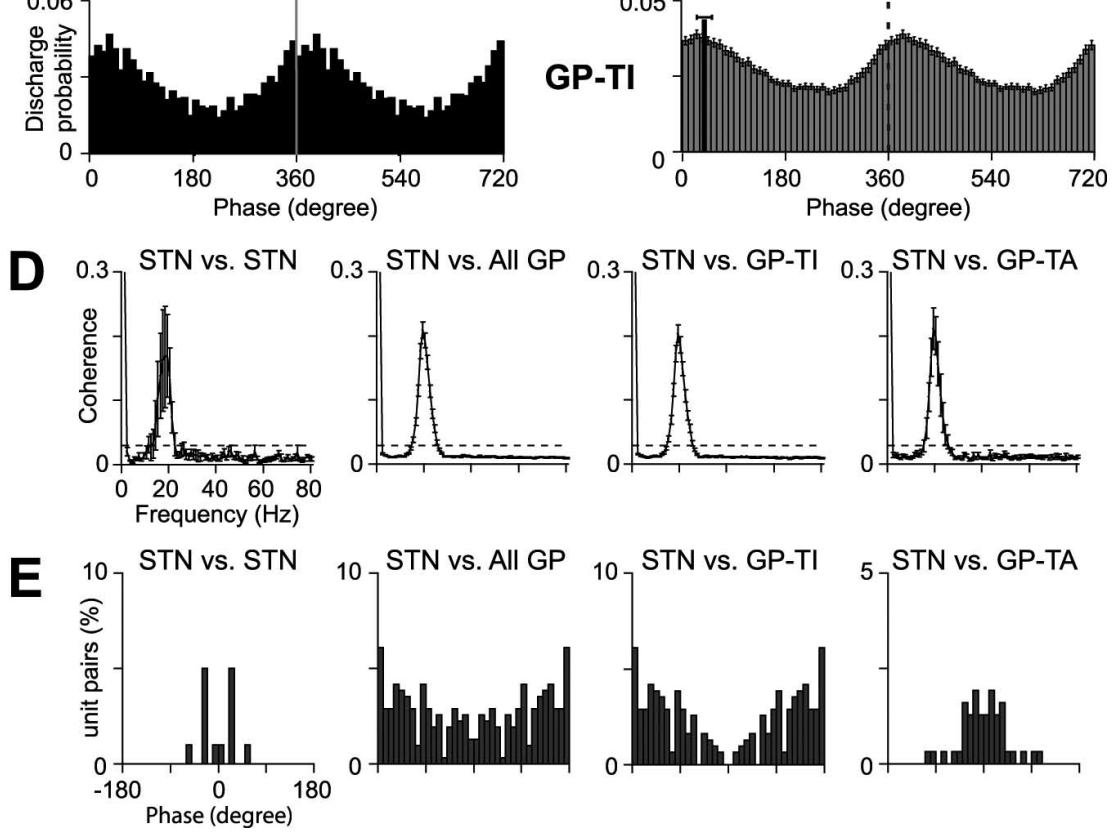

Figure 7. Spike timing of subthalamic nucleus and globus pallidus neurons in relation to cortical $\beta$ oscillations in 6-OHDAlesioned rats. $\boldsymbol{A}$, Simultaneous recording of a single STN neuron and two GP neurons of different types (GP-TA and GP-TI) during cortical $\beta$ oscillations (see $\mathrm{E}(\mathrm{OG})$ in a lesioned rat. Gray lines centered on peaks of the cortical $\beta$ oscillation exemplify spike timing relationships. Calibration: $100 \mu \mathrm{V}$ (filtered ECoG), $500 \mu \mathrm{V}$ (STN unit), and $200 \mu \mathrm{V}$ (GP units). B, Cross-correlograms (CC) show oscillatory synchronization of the STN versus GP-TA neuron pair and STN versus GP-TI pair at $\beta$ frequencies $(\sim 20 \mathrm{~Hz})$. C, Linear phase histograms for neurons in $\boldsymbol{A}$ (left) and for all tested STN neurons, GP-TA neurons, and GP-TI neurons (right). For clarity, two cortical $\beta$ oscillation cycles are shown. Data in population histograms (right) are means \pm 1 SEM. The vertical black lines indicate the mean phase angles (error bars are 2 SEM). D, Mean coherence for all STN versus STN unit pairs, STN versus all GP unit pairs, STN versus GP-TI unit pairs, and STN versus GP-TA unit pairs ( $p=0.05$, dashed lines). $\boldsymbol{E}$, Plots of instantaneous phase values for all pairs of neurons shown in $\boldsymbol{D}$.

\section{Discussion}

Excessive $\beta$ oscillations (15-30 Hz) emerge in corticobasal ganglia circuits involving the STN in PD. Here, we resolve several critical issues surrounding the cellular and network substrates of these pathological oscillations. We demonstrate using large-scale recordings that oscillatory activity in GP neuronal networks also becomes excessively and selectively synchronized at $\beta$ frequencies in a spatially widespread and brain statedependent manner after dopamine loss. We also show that GP contains two types of neuron with distinct temporal couplings, firing rates and patterns that are maintained across extreme brain states. Finally, the precisely timed discharges of GP and STN neurons indicate that rhythmic sequences of recurrent excitation and inhibition in the STN-GP network, and lateral inhibition between GP neurons, could support abnormal $\beta$ oscillations. We propose that GP neurons are critical for orchestrating exaggerated $\beta$ oscillations in STN and corticobasal ganglia circuits in PD.

Organization of abnormal neuronal activity after dopamine loss

Our recordings of STN-GP network activity in control and lesioned rats during SWA and cortical activation elucidated common organizational principles of function and dysfunction. First, dopamine loss is associated with hypoactivity in GP and hyperactivity in STN. Second, these firing rate changes are accompanied by profound increases in synchronized oscillatory activity. The predominant frequency of abnormal synchronized oscillations was dependent on brain state. During SWA, $\sim 1 \mathrm{~Hz}$ synchronization prevailed, while during cortical activation, $\beta$ oscillations were selectively exaggerated. Our data thus reconcile two divergent models of BG dysfunction that account for parkinsonian symptoms on the basis of changes in firing rate (DeLong, 1990) or firing pattern, including excessive synchronization (Bergman et al., 1998). Activity disturbances in time and frequency domains are likely both important determinants of parkinsonian phenotypes. Although the quantitative impact of each factor on information processing remains unclear, our results suggest that the relative contributions of changes in firing rate, firing regularity and synchronization may depend on the nucleus (GP vs STN) or even neuron type within a nucleus. With respect to the latter, we also identified two types of GP neuron: GP-TA neurons preferentially discharged during the active components of ongoing cortical slow oscillations, thus more faithfully representing rhythmic STN outputs; conversely, GP-TI neurons preferentially discharged during inactive components, thus better reflecting rhythmic striatal outputs (Mallet et al., 2006; Zold et al., 2007). Because the two types 
of GP neuron tended to fire in anti-phase, local lateral inhibition could also partly underlie these firing preferences (Sadek et al., 2007). This functional dichotomy was largely preserved across extreme brain states in lesioned animals. Thus, ensembles containing the same GP neuron type tended to consistently fire inphase, whereas mixed ensembles comprised of GP-TA and GP-TI neurons persistently fired in anti-phase. However, brain state transitions are commonly accompanied by a rapid reorganization of ensembles in space and time (Somogyi and Klausberger, 2005; Magill et al., 2006). Unusually then, functionally distinct GP ensembles are not completely reorganized, but rather, their interactions are largely governed by "hard wiring." Interestingly, two GP neuron types, low-frequency discharge "bursters" and high-frequency discharge "pausers," exist in awake monkeys (DeLong, 1971). Whether these neurons are related to GP-TA and GP-TI neurons should be established.

\section{STN-GP network interactions during excessive $\boldsymbol{\beta}$ oscillations}

Phase histograms of unit activity during $\beta$ oscillations showed that, on average, the maximal firing of GP-TA and GP-TI neurons lagged that of STN neurons by 22 and 153 degrees, respectively. Given a mean $\beta$ oscillation period of $\sim 50 \mathrm{~ms}$, these phase lags represent time delays of $\sim 3 \mathrm{~ms}$ and $\sim 21 \mathrm{~ms}$, which tally with the mean latency and time-to-peak, respectively, of monosynaptic subthalamic EPSPs evoked in GP (Kita and Kitai, 1991). All GP neurons likely receive STN input and emit local axon collaterals (Smith et al., 1998; Sadek et al., 2007). So, what underlies this timing difference of GP-TA and GP-TI neuron discharges? More effective driving of GP-TA neurons by rhythmic STN output, and then feed-forward inhibition of GP-TI neurons by GP-TA neurons could be a factor in delaying activity of GP-TI neurons. Our SWA recordings suggest that GP-TI neurons are more sensitive to rhythmic striatal outputs, which could also contribute. Moreover, GP-TI neurons could be hyperpolarized further from their firing threshold. In support of this, GP-TA neurons fired at significantly higher rates than GP-TI neurons. Thus, timing differences could be a consequence of disparate innervation of GP neurons by STN/striatum and/or distinct intrinsic membrane properties. Regardless of these differences, lateral inhibition between GP neurons could shape pallidal activity during $\beta$ oscillations, and GABAergic feed-back from both types of GP neuron could subsequently inhibit and sculpt oscillatory activity in STN. Indeed, STN neuron firing decreased when GP neurons were most active. Single GP neurons sparsely innervate the STN (M. Bevan, personal communication). Thus, relatively large GP ensembles must be synchronized to widely synchronize STN neurons. During excessive $\beta$ oscillations, synchronized STN outputs likely drive widespread synchronized activity in GP ensembles. Rhythmic, synchronized feed-back inhibition from GP could summate across, and synchronize, STN neurons, as well as increase the impact of any rhythmic excitatory inputs (Baufreton et al., 2005). Subsequent reverberation of synchronized activity in the reciprocally connected STN-GP network would further recruit neurons into $\beta$ oscillations.

Dichotomous feed-forward/feed-back interactions between neurons within an inappropriately synchronized and oscillatory STN-GP network may not only emerge after dopamine loss. Indeed, a similar functional organization arises during the synchronous spike-and-wave rhythms $(7-10 \mathrm{~Hz})$ accompanying absence epileptic seizures ( $\mathrm{Paz}$ et al., 2005), indicating that analogous mechanisms could be unleashed and underlie distinct pathological oscillations in these circuits. Emphasis has often been placed on the importance of emergent pathological "bursting" activity in the BG in PD (Bergman et al., 1994; Bevan et al., 2002). Recordings in organotypic cocultures suggest that a bursting STN-GP network can alone support oscillations with frequencies of $\leq 5 \mathrm{~Hz}$ (Plenz and Kital, 1999). However, during any given $\beta$ oscillation cycle, most GP neurons did not burst but instead fired a single spike in a precisely timed manner. Whether the STN-GP network is a central pattern generator for $\beta$ oscillations in vivo is uncertain. While it is conceptually appealing to "start" a hypothetical $\beta$ oscillation with a barrage of synchronous STN outputs, it is difficult to determine which structure(s) initiate these excessive rhythms because they likely stem from the emergent properties of the reciprocally connected network itself. Although STN neurons are intrinsic pacemakers (Bevan et al., 2006), the stereotypical unit activity we recorded during $\beta$ oscillations has not been described exvivo. Thus, while changes in the intrinsic membrane properties of STN neurons cannot be disregarded, it seems likely that intact afferents are critical for instigating or orchestrating excessively synchronized outputs from STN. The abnormal slow oscillations generated in the STN-GP network during SWA are driven by cortex (Magill et al., 2001) and this may apply to abnormal $\beta$ oscillations. Indeed, field potentials recorded in ipsilateral frontal cortex, which projects directly to STN/striatum (Smith et al., 1998), also displayed excessive $\beta$ oscillations of similar frequencies to those in STN/GP. Thus, synchronized cortical inputs are a candidate for recruiting STN neurons into population $\beta$ rhythms (Magill et al., 2004). Because striatum will likely receive rhythmic cortical inputs, striatopallidal inhibition may also be important for the $\beta$ oscillations in GP. Cortex recruits STN neurons at much shorter latencies than striatal projection neurons, effectively staggering the arrival of subthalamic and striatal inputs in GP by 10-15 ms (Magill et al., 2004; Mallet et al., 2006). If rhythmic striatopallidal inputs dominated, the probability of GP firing should be minimal from 10 to $15 \mathrm{~ms}$ after STN firing. However, this was not the case for most GP neurons. Thus, precisely timed striatal outputs may not play a dominant role in actively orchestrating the excessive $\beta$ oscillations that emerge in the STN-GP network. Increased striatopallidal neuron activity after dopamine loss however (Mallet et al., 2006) might partly account for the overall decrease in GP neuron firing during $\beta$ oscillations.

\section{Further functional implications}

Dopamine coordinates neuronal activity in the frequency domain (Costa et al., 2006). When controlled by dopamine, dynamic $\beta$ oscillations in corticobasal ganglia circuits may be important for normal movement (Courtemanche et al., 2003; Rubino et al., 2006). However, after dopamine loss, inappropriate reverberatory interactions within GP, and between GP and STN, may not only support but also actively promote the emergence of excessively synchronized $\beta$ oscillations at the network level. Indeed, cycles of precisely timed excitatory and inhibitory outputs from STN and GP, respectively, could synchronize their common targets and thus, the excessive rhythms propagate to BG output nuclei (Brown, 2006) and thence, thalamocortical and midbrain circuits. Therefore, GP neurons, by virtue of their widespread innervation of all BG nuclei and feed-back/feed-forward mechanisms, are in a central position to orchestrate the generation and propagation of exaggerated $\beta$ oscillations in STN and the entire BG. Precisely if and how the altered firing rates/patterns we define here impact on behavior is uncertain. However, dysregulation of corticobasal ganglia network activity in space and time after dopamine loss will profoundly affect information processing therein (Mallet et al., 2008), providing a possible 
mechanism whereby excessive $\beta$ oscillations may be pathological in PD (Brown, 2006).

\section{References}

Bar-Gad I, Ritov Y, Vaadia E, Bergman H (2001) Failure in identification of overlapping spikes from multiple neuron activity causes artificial correlations. J Neurosci Methods 107:1-13.

Bar-Gad I, Heimer G, Ritov Y, Bergman H (2003) Functional correlations between neighboring neurons in the primate globus pallidus are weak or nonexistent. J Neurosci 23:4012-4016.

Barthó P, Hirase H, Monconduit L, Zugaro M, Harris KD, Buzsáki G (2004) Characterization of neocortical principal cells and interneurons by network interactions and extracellular features. J Neurophysiol 92:600-608.

Baufreton J, Atherton JF, Surmeier DJ, Bevan MD (2005) Enhancement of excitatory synaptic integration by GABAergic inhibition in the subthalamic nucleus. J Neurosci 25:8505-8517.

Belluscio MA, Kasanetz F, Riquelme LA, Murer MG (2003) Spreading of slow cortical rhythms to the basal ganglia output nuclei in rats with nigrostriatal lesions. Eur J Neurosci 17:1046-1052.

Bergman H, Wichmann T, Karmon B, DeLong MR (1994) The primate subthalamic nucleus. II. Neuronal activity in the MPTP model of parkinsonism. J Neurophysiol 72:507-520.

Bergman H, Feingold A, Nini A, Raz A, Slovin H, Abeles M, Vaadia E (1998) Physiological aspects of information processing in the basal ganglia of normal and parkinsonian primates. Trends Neurosci 21:32-38.

Bevan MD, Magill PJ, Terman D, Bolam JP, Wilson CJ (2002) Move to the rhythm: oscillations in the subthalamic nucleus-external globus pallidus network. Trends Neurosci 25:525-531.

Bevan MD, Atherton JF, Baufreton J (2006) Cellular principles underlying normal and pathological activity in the subthalamic nucleus. Curr Opin Neurobiol 16:621-628.

Bolam JP, Hanley JJ, Booth PA, Bevan MD (2000) Synaptic organisation of the basal ganglia. J Anat 196:527-542.

Boraud T, Brown P, Goldberg JA, Graybiel AM, Magill PJ (2005) Oscillations in the basal ganglia: the good, the bad and the unexpected. In: The basal ganglia VIII (Bolam JP, Ingham CA, Magill PJ, eds), pp 3-24. New York: Springer Science and Business Media.

Brown P (2006) Bad oscillations in Parkinson's disease. J Neural Transm Suppl 70:27-30.

Brown P, Oliviero A, Mazzone P, Insola A, Tonali P, Di Lazzaro V (2001) Dopamine dependency of oscillations between subthalamic nucleus and pallidum in Parkinson's disease. J Neurosci 21:1033-1038.

Buzsáki G, Draguhn A (2004) Neuronal oscillations in cortical networks. Science 304:1926-1929.

Costa RM, Lin SC, Sotnikova TD, Cyr M, Gainetdinov RR, Caron MG, Nicolelis MA (2006) Rapid alterations in corticostriatal ensemble coordination during acute dopamine-dependent motor dysfunction. Neuron 52:359-369.

Courtemanche R, Fujii N, Graybiel AM (2003) Synchronous, focally modulated beta-band oscillations characterize local field potential activity in the striatum of awake behaving monkeys. J Neurosci 23:11741-11752.

DeLong MR (1971) Activity of pallidal neurons during movement. J Neurophysiol 34:414-427.

DeLong MR (1990) Primate models of movement disorders of basal ganglia origin. Trends Neurosci 13:281-285.

Engel AK, Fries P, Singer W (2001) Dynamic predictions: oscillations and synchrony in top-down processing. Nat Rev Neurosci 2:704-716.

Gatev P, Darbin O, Wichmann T (2006) Oscillations in the basal ganglia under normal conditions and in movement disorders. Mov Disord 21:1566-1577.

Halliday DM, Rosenberg JR, Amjad AM, Breeze P, Conway BA, Farmer SF (1995) A framework for the analysis of mixed time series/point process data-theory and application to the study of physiological tremor, single motor unit discharges and electromyograms. Prog Biophys Mol Biol 64:237-278

Hammond C, Bergman H, Brown P (2007) Pathological synchronization in Parkinson's disease: networks, models and treatments. Trends Neurosci 30:357-364

Johnson DH (1996) Point process models of single-neuron discharges. J Comput Neurosci 3:275-299.

Kita H, Kitai ST (1991) Intracellular study of rat globus pallidus neurons: membrane properties and responses to neostriatal, subthalamic and nigral stimulation. Brain Res 564:296-305.

Kita H, Kitai ST (1994) The morphology of globus pallidus projection neurons in the rat: an intracellular staining study. Brain Res 636:308-319.

Kühn AA, Trottenberg T, Kivi A, Kupsch A, Schneider GH, Brown P (2005) The relationship between local field potential and neuronal discharge in the subthalamic nucleus of patients with Parkinson's disease. Exp Neurol 194:212-220.

Levy R, Ashby P, Hutchison WD, Lang AE, Lozano AM, Dostrovsky JO (2002) Dependence of subthalamic nucleus oscillations on movement and dopamine in Parkinson's disease. Brain 125:1196-1209.

Magill PJ, Bolam JP, Bevan MD (2001) Dopamine regulates the impact of the cerebral cortex on the subthalamic nucleus-globus pallidus network. Neuroscience 106:313-330.

Magill PJ, Sharott A, Bevan MD, Brown P, Bolam JP (2004) Synchronous unit activity and local field potentials evoked in the subthalamic nucleus by cortical stimulation. J Neurophysiol 92:700-714.

Magill PJ, Pogosyan A, Sharott A, Csicsvari J, Bolam JP, Brown P (2006) Changes in functional connectivity within the rat striatopallidal axis during global brain activation in vivo. J Neurosci 26:6318-6329.

Mallet N, Le Moine C, Charpier S, Gonon F (2005) Feedforward inhibition of projection neurons by fast-spiking GABA interneurons in the rat striatum in vivo. J Neurosci 25:3857-3869.

Mallet N, Ballion B, Le Moine C, Gonon F (2006) Cortical inputs and GABA interneurons imbalance projection neurons in the striatum of parkinsonian rats. J Neurosci 26:3875-3884.

Mallet N, Pogosyan A, Sharott A, Csicsvari J, Bolam JP, Brown P, Magill PJ (2008) Disrupted dopamine transmission and the emergence of exaggerated beta oscillations in subthalamic nucleus and cerebral cortex. J Neurosci 28:4795-4806.

Mitzdorf U (1985) Current source-density method and application in cat cerebral cortex: investigation of evoked potentials and EEG phenomena. Physiol Rev 65:37-100.

Nambu A, Llinaś R (1994) Electrophysiology of globus pallidus neurons in vitro. J Neurophysiol 72:1127-1139.

Nini A, Feingold A, Slovin H, Bergman H (1995) Neurons in the globus pallidus do not show correlated activity in the normal monkey, but phaselocked oscillations appear in the MPTP model of parkinsonism. J Neurophysiol 74:1800-1805.

Oorschot DE (1996) Total number of neurons in the neostriatal, pallidal, subthalamic, and substantia nigral nuclei of the rat basal ganglia: a stereological study using the cavalieri and optical disector methods. J Comp Neurol 366:580-599.

Paxinos G, Watson C (1986) The rat brain in stereotaxic coordinates, Ed 2. Sydney: Academic.

Paz JT, Deniau JM, Charpier S (2005) Rhythmic bursting in the corticosubthalamo-pallidal network during spontaneous genetically determined spike and wave discharges. J Neurosci 25:2092-2101.

Plenz D, Kital ST (1999) A basal ganglia pacemaker formed by the subthalamic nucleus and external globus pallidus. Nature 400:677-682.

Rubino D, Robbins KA, Hatsopoulos NG (2006) Propagating waves mediate information transfer in the motor cortex. Nat Neurosci 9:1549-1557.

Sadek AR, Magill PJ, Bolam JP (2007) A single-cell analysis of intrinsic connectivity in the rat globus pallidus. J Neurosci 27:6352-6362.

Schnitzler A, Gross J (2005) Normal and pathological oscillatory communication in the brain. Nat Rev Neurosci 6:285-296.

Schwarting RK, Huston JP (1996a) Unilateral 6-hydroxydopamine lesions of meso-striatal dopamine neurons and their physiological sequelae. Prog Neurobiol 49:215-266.

Schwarting RK, Huston JP (1996b) The unilateral 6-hydroxydopamine lesion model in behavioral brain research. Analysis of functional deficits, recovery and treatments. Prog Neurobiol 50:275-331.

Sharott A, Magill PJ, Harnack D, Kupsch A, Meissner W, Brown P (2005) Dopamine depletion increases the power and coherence of betaoscillations in the cerebral cortex and subthalamic nucleus of the awake rat. Eur J Neurosci 21:1413-1422.

Singer W (1999) Neuronal synchrony: a versatile code for the definition of relations? Neuron 24:49-65, 111-125.

Smith Y, Bevan MD, Shink E, Bolam JP (1998) Microcircuitry of the direct and indirect pathways of the basal ganglia. Neuroscience 86:353-387. 
Somogyi P, Klausberger T (2005) Defined types of cortical interneurone structure space and spike timing in the hippocampus. J Physiol 562:9-26.

Stanford IM (2003) Independent neuronal oscillators of the rat globus pallidus. J Neurophysiol 89:1713-1717.

Steriade M (2000) Corticothalamic resonance, states of vigilance and mentation. Neuroscience 101:243-276.

Surmeier DJ, Mercer JN, Chan CS (2005) Autonomous pacemakers in the basal ganglia: who needs excitatory synapses anyway? Curr Opin Neurobiol 15:312-318.

Terman D, Rubin JE, Yew AC, Wilson CJ (2002) Activity patterns in a model for the subthalamopallidal network of the basal ganglia. J Neurosci 22:2963-2976.

Uhlhaas PJ, Singer W (2006) Neural synchrony in brain disorders: relevance for cognitive dysfunctions and pathophysiology. Neuron 52:155-168.

Vila M, Périer C, Féger J, Yelnik J, Faucheux B, Ruberg M, Raisman-Vozari R, Agid Y, Hirsch EC (2000) Evolution of changes in neuronal activity in the subthalamic nucleus of rats with unilateral lesion of the substantia nigra assessed by metabolic and electrophysiological measurements. Eur J Neurosci 12:337-344.

Walters JR, Hu D, Itoga CA, Parr-Brownlie LC, Bergstrom DA (2007) Phase relationships support a role for coordinated activity in the indirect pathway in organizing slow oscillations in basal ganglia output after loss of dopamine. Neuroscience 144:762-776.

Williams D, Tijssen M, Van Bruggen G, Bosch A, Insola A, Di Lazzaro V, Mazzone P, Oliviero A, Quartarone A, Speelman H, Brown P (2002) Dopamine-dependent changes in the functional connectivity between basal ganglia and cerebral cortex in humans. Brain 125:1558-1569.

Williams D, Kühn A, Kupsch A, Tijssen M, van Bruggen G, Speelman H, Hotton G, Loukas C, Brown P (2005) The relationship between oscillatory activity and motor reaction time in the parkinsonian subthalamic nucleus. Eur J Neurosci 21:249-258.

Zold CL, Ballion B, Riquelme LA, Gonon F, Murer MG (2007) Nigrostriatal lesion induces D2-modulated phase-locked activity in the basal ganglia of rats. Eur J Neurosci 25:2131-2144. 